Journal of Scientific Computing, 2004, to appear.

\title{
BOUNDARY LAYERS IN AN OCEAN RELATED SYSTEM
}

\author{
A. ROUSSEAU ${ }^{b}$, R. TEMAM ${ }^{b *}$, AND J. TRIBBIA $^{\sharp}$ \\ b'Laboratoire d'Analyse Numérique, Université Paris-Sud, Orsay, France. \\ *The Institute for Scientific Computing and Applied Mathematics, \\ Indiana University, Bloomington, IN, USA. \\ ${ }^{\sharp}$ National Center for Atmospheric Research, Boulder, Colorado, USA.
}

\begin{abstract}
In this article two regularizations of an hyperbolic model system derived from the primitive equations of the ocean (or the atmosphere) are presented. The two regularized systems converge to different limits as the regularization parameter converges to 0 . Numerical approximations of these equations and numerical simulations are also presented.
\end{abstract}

\section{INTRODUCTION}

The Primitive Equations of the ocean and the atmosphere are fundamental equations of geophysical fluid mechanics $([9],[14])$. In the presence of viscosity, it has been shown - in various contexts - that these equations are well-posed (see e.g. [6], [7], and the review article [13]).

In the absence of viscosity, it is known that the PEs are not well-posed for any set of boundary conditions of local type. This difficulty is analyzed in [8] using a modal analysis in the vertical direction (see also [12]). To overcome this difficulty a modification of the PEs was proposed in [12], for which a set of local boundary conditions also given in [12] produces a decay of energy (in the absence of forcing), an important positive step in proving well-posedness. The modified PEs introduced in [12] contain an added friction term $\delta w$ in the hydrostatic equation, so that the model is actually nonhydrostatic ; we will call this system the $\delta$-PEs equations.

A first objective of this work was to study the $\delta$-PEs equations linearized around a flow of constant velocity $U_{0}$ in direction $(O x)$. For that purpose we considered

Date: September 17, 2007.

Key words and phrases. Primitive Equations of the Ocean, Boundary Layers, Correctors, Transparent Boundary Conditions, Finite Element Method. 
an expansion of the solutions using suitable normal modes in the vertical direction, thus leading to a system of three equations (see below for more details) :

$$
\left\{\begin{array}{l}
u_{t}-f v+\phi_{x}-\frac{\delta g}{N^{2} \lambda^{2}} u_{x x}+U_{0} u_{x}=0 \\
v_{t}+f u+U_{0} v_{x}=0 \\
\phi_{t}+U_{0} \phi_{x}+\frac{1}{\lambda^{2}} u_{x}=0 .
\end{array}\right.
$$

This system is supplemented with suitable boundary conditions described below (see (2.3)) and we were interested in finding the limit of the solutions of this system as $\delta \rightarrow 0$ (or $\varepsilon \rightarrow 0, \varepsilon=\delta g / 2 N^{2} \lambda^{2}$ ). Actually, we were surprised to learn that the limit system was not what one could believe at first. Furthermore, we found the boundary conditions for the limit system thanks to numerical simulations, by inspection of the solutions to system (1.1) (see also (1.8)) for small $\varepsilon$. Subsequent theorical studies, to be reported in [10], confirmed that the boundary conditions of the limit system were those hinted at by numerical simulations. The latter were done using a numerical procedure described in Section 2.3 and Remark 2.3.

Depending on the problem that we study, the limit system for (1.1) (or (1.8)) provided in Section 2 may or may not be suitable. In Section 3 we propose another version of system (1.8) (equations and boundary conditions), inspired by the concept of transparent boundary conditions. In that case, the limit system is the same as before (one mode of the nonviscous PEs) as $\varepsilon \rightarrow 0$, but the boundary conditions are different, somehow more natural. We realize of course that deciding what are the "good" boundary conditions for the limit system is a matter of physical intuition and may depend on the physical problem considered. For the problem of the PEs studied in [8] and [12], the $\delta$-PEs are supplemented with boundary conditions of local type, but this modal analysis suggests that the model produces boundary layers and some undesirable reflection of waves at the boundary. These difficulties disappear in the TBC (transparent boundary condition) model studied in Section 3. However, passing from one mode to the whole PEs, this study suggests that nonlocal (mode dependant) boundary conditions and implicit or semi-implicit procedures are required for the TBC approach, whereas easier local boundary conditions and fully explicit schemes are available for the $\delta$-PEs. Finally, the choice will depend on the objectives, that is the desired precision versus the amount of computational effort that one wishes to invest. In the case of the PEs this study will be conducted elsewhere.

We conclude this section by recalling the derivation of system (1.1)-(1.8) studied in Section 2.

We start from the primitive equations of the ocean without viscosity : 


$$
\left\{\begin{array}{c}
\frac{\partial \mathbf{v}}{\partial t}+(\mathbf{v} \cdot \nabla) \mathbf{v}+w \frac{\partial \mathbf{v}}{\partial z}+f \mathbf{k} \times \mathbf{v}+\nabla \phi=0 \\
\delta w+\frac{\partial p}{\partial z}=-\rho g \\
\nabla \cdot \mathbf{v}+\frac{\partial w}{\partial z}=0 \\
\frac{\partial \theta}{\partial t}+(\mathbf{v} \cdot \nabla) \theta+w \frac{\partial \theta}{\partial z}=0 \\
\rho=\rho(\theta)
\end{array}\right.
$$

In these equations $\mathbf{v}=(u, v)$ is the horizontal velocity, $w$ the vertical velocity, $\phi$ the pressure, $\rho$ the density, and $\theta$ the temperature. $g$ is the gravitational acceleration, and $f$ the Coriolis parameter. The horizontal gradient is denoted by $\nabla$.

Here $\delta \geq 0$; in the original version of the PEs, $\delta=0$, and for $\delta>0$ we obtain the $\delta$-PEs considered in [12] (see also [11] in a different context).

A linearization of (1.2) around a constant flow velocity $U_{0}$ in the $x$ direction $\left(U_{0}>0\right)$, with no dependance in $y$ reads :

$$
\left\{\begin{array}{l}
u_{t}-f v+\phi_{x}+U_{0} u_{x}=0 \\
v_{t}+f u+U_{0} v_{x}=0 \\
\theta_{t}+N^{2} \frac{\theta_{0}}{g} w+U_{0} \theta_{x}=0 \\
u_{x}+w_{z}=0 \\
\delta w+\phi_{z}=\frac{g \theta}{\theta_{0}}
\end{array}\right.
$$

where $N$ is the Brunt-Väisälä (or buoyancy) frequency, supposed constant.

The boundary conditions proposed in [12] for the $\delta$-system (1.2) induce the following boundary conditions for (1.3) (valid under the hypothesis that $u$ is small compared with $\left.U_{0}\right)$ :

$$
\left\{\begin{array}{lll}
u(0, z, t)=u_{g l}(z, t), & u(L, z, t)=u_{g r}(z, t), & z \in[-H, 0], \quad t>0 \\
v(0, z, t)=v_{g l}(z, t), & \theta(0, z, t)=\theta_{g l}(z, t), & z \in[-H, 0], \quad t>0 \\
w(x,-H, t)=0, & w(x, 0, t)=0, & x \in[0, L], \quad t>0
\end{array}\right.
$$

with $\int_{-H}^{0} u_{g l}(z, t) d z=\int_{-H}^{0} u_{g r}(z, t) d z$, so that we can integrate the 4 th equation of (1.3) and guarantee that $w=0$ at $z=-H$ and 0 .

The method used in [12] to show the decay of energy for the $\delta$-PEs extends to system (1.3)-(1.4) in the following way. We assume here for simplicity that $u_{g l}, u_{g r}, v_{g l}$ and $\theta_{g l}$ vanish ; the treatment of the nonhomogeneous boundary conditions will be explained below for our actual equations (see Remark 2.2). For an arbitrary $\kappa>0$, 
we multiply (1.3a) by $u,(1.3 \mathrm{~b})$ by $v,(1.3 \mathrm{c})$ by $\kappa \theta$ and (1.3e) by $w$, integrate over $\mathcal{M}=(0, L) \times(-H, 0)$ and add these relations. We find

$$
\frac{d}{d t} \int_{\mathcal{M}}\left(u^{2}+v^{2}+\kappa \theta^{2}\right) d \mathcal{M}+2 \delta \int_{\mathcal{M}} w^{2} d \mathcal{M}+I_{1}+I_{2}+I_{3}=I_{4}
$$

where,

$$
\left\{\begin{array}{l}
I_{1}=2 \int_{\mathcal{M}}\left(\phi_{x} u+\phi_{z} w\right) d \mathcal{M} \\
I_{2}=U_{0} \int_{-H}^{0}\left\{\left[u^{2}(x, z)+v^{2}(x, z)+\kappa \theta^{2}(x, z)\right]_{x=0}^{x=L}\right\} d z \\
I_{3}=2 \kappa N^{2} \int_{\mathcal{M}} \frac{\theta_{0} \theta}{g} w d \mathcal{M} \\
I_{4}=2 \int_{\mathcal{M}} \frac{g \theta}{\theta_{0}} w d \mathcal{M} .
\end{array}\right.
$$

Setting $\kappa=\left(g / N \theta_{0}\right)^{2}$, we find $I_{3}=I_{4}$, and these terms cancel each other in $(1.5)$; it is then easy to check with $(1.3 \mathrm{~d})$ and (1.4) that $I_{1}=0, I_{2} \geq 0$. That is, (1.5) ensures that the energy $E_{\kappa}(t)=\int_{\mathcal{M}}\left(u^{2}+v^{2}+\kappa \theta^{2}\right) d \mathcal{M}$ is time-decreasing (for $\left.\kappa=\left(g / N \theta_{0}\right)^{2}\right)$, which is an essential step in the proof of well-posedness of the linear system (1.3)-(1.4) (see [5] or [15] for more details).

In [8] and [12], a mode analysis of system (1.3) in the $z$ direction was implemented; the authors proceed by separations of variables when looking for a solution of (1.3). They search $u$ and the other variables of the form $u(x, z, t)=U(z) \hat{u}(x, t), v(x, z, t)=$ $U(z) \hat{v}(x, t), \phi(x, z, t)=U(z) \hat{\phi}(x, t)$. Substituting these expressions into the equations, and combining them together we obtain the following modal equation (again, see [8] or [12] for the definition of the function $U(z)$, and more details) :

$$
\left\{\begin{array}{l}
\hat{u}_{n t}-f \hat{v}_{n}+\hat{\phi}_{n x}+U_{0} \hat{u}_{n x}=0, \\
\hat{v}_{n t}+f \hat{u}_{n}+U_{0} \hat{v}_{n x}=0, \\
\hat{\phi}_{n t}+U_{0} \hat{\phi}_{n x}+\frac{1}{\left(\lambda_{n}\right)^{2}} \hat{u}_{n x}=0,
\end{array} \text { for the } n\right. \text {th mode. }
$$

The characteristic values of (1.7) - determined by the first order parts - are $U_{0}$, $U_{0}+\lambda^{-1}$ and $U_{0}-\lambda^{-1}\left(\lambda=\lambda_{n}\right)$. Hence, since the domain is $(0, L)$ in the $x$ direction, if $\lambda^{-1}<U_{0}$ three boundary conditions are needed at $x=0$. Conversely, if $\lambda^{-1}>U_{0}$, only two boundary conditions are needed at $x=0$, and one is needed at $x=L$. This is why the global problem is ill-posed for a local set of boundary conditions. The study below focuses on the most problematical case, $\lambda^{-1}>U_{0}$, corresponding to the so called subcritical modes. 
For notational convenience, we now write $u, v, \phi$ and $\lambda$ instead of $\tilde{u}_{n}, \tilde{v}_{n}, \tilde{\phi}_{n}$ and $\lambda_{n}$. With $\delta>0$, the same study leads to the following system, which naturally reduces to (1.7) if $\delta=0$ :

$$
\left\{\begin{array}{l}
u_{t}-f v+\phi_{x}-\frac{\delta g}{N^{2} \lambda^{2}} u_{x x}+U_{0} u_{x}=0 \\
v_{t}+f u+U_{0} v_{x}=0 \\
\phi_{t}+U_{0} \phi_{x}+\frac{1}{\lambda^{2}} u_{x}=0 .
\end{array}\right.
$$

From (1.4), one can derive, after some approximations, the following boundary conditions for system (1.8) :

$$
\left\{\begin{array}{ll}
u(0, t)=u_{g l}(t), & u(L, t)=u_{g r}(t), \\
v(0, t)=v_{g l}(t), & \phi(0, t)=\phi_{g l}(t),
\end{array} \quad t>0\right.
$$

From now on, we set $f=0$ since the Coriolis effect is not an essential element in the well-posedness issue. With this simplification, the second equation (1.8) can be solved independently of the other equations, and we can study the system (1.8),(1.9), in which the $v$ variable is now omitted.

2. One mode AnAlysis of the $\delta$-PEs.

\subsection{The equations and boundary condition.}

With the simplification $f=0$, we can now focus on the equations coupling $u$ and $\phi:$

$$
\begin{gathered}
\left\{\begin{array}{l}
u_{t}+\phi_{x}-2 \varepsilon u_{x x}+U_{0} u_{x}=F_{u}, \\
\phi_{t}+U_{0} \phi_{x}+\frac{1}{\lambda^{2}} u_{x}=F_{\phi} .
\end{array}\right. \\
\left\{\begin{array}{l}
u(0, t)=u_{g l}(t), u(L, t)=u_{g r}(t), \\
\phi(0, t)=\phi_{g l}(t),
\end{array}\right.
\end{gathered}
$$

In (2.1) we have introduced for mathematical generality the source terms $F_{u}$ and $F_{\phi}$, which do not exist in the original problem ; we also set $\varepsilon=\delta g / 2 N^{2} \lambda^{2}$, which thus depends on $\lambda=\lambda_{n}$. Using a change of variable suggested by the diagonalization of the limit system $((2.1)$ with $\varepsilon=0$, see below $)$, we set $\xi=u+\lambda \phi$ and $\eta=u-\lambda \phi$. The system (2.1) becomes:

$$
\left\{\begin{array}{l}
\xi_{t}+\alpha \xi_{x}-\varepsilon\left(\xi_{x x}+\eta_{x x}\right)=f_{\xi} \\
\eta_{t}-\beta \eta_{x}-\varepsilon\left(\xi_{x x}+\eta_{x x}\right)=f_{\eta}
\end{array}\right.
$$


where

$$
\left\{\begin{array}{l}
\alpha=U_{0}+\frac{1}{\lambda}>0 \\
\beta=-U_{0}+\frac{1}{\lambda} .
\end{array}\right.
$$

In the two following sections, we will study the system (2.3) with two different sets of boundary conditions. From now on we will also suppose $\beta>0$, i.e. $U_{0}<\lambda^{-1}$ which, as we said, is the most problematic case in the physical problem (subcritical modes).

\section{Boundary conditions}

We are interested in studying the system (2.3), with the Dirichlet boundary conditions proposed in [12].

For system (2.1) the natural boundary conditions are as follows :

$$
\left\{\begin{array}{l}
u(0, t)=u_{g l}(t), u(L, t)=u_{g r}(t), \quad t>0 . \\
\phi(0, t)=\phi_{g l}(t),
\end{array}\right.
$$

In the $(\xi, \eta)$ variables, the boundary conditions (2.2) yield the following boundary conditions for the system (2.3) :

$$
\left\{\begin{array}{l}
\xi(0, t)=\xi_{g l}(t), \eta(0, t)=\eta_{g l}(t), \quad t>0 \\
(\xi+\eta)(L, t)=2 u_{g r}(t)
\end{array}\right.
$$

where we remember that $\xi+\eta=2 u$, and set $\xi_{g l}(t)=u_{g l}(t)+\lambda \phi_{g l}(t), \eta_{g l}(t)=$ $u_{g l}(t)-\lambda \phi_{g l}(t)$.

\section{The limit system}

We discuss the (formal) passage to the limit $\varepsilon \rightarrow 0$ in the homogeneous boundary condition case, where $u_{g l}, u_{g r}$ and $\phi_{g l}$ vanish. Setting formally $\varepsilon=0$ in (2.1) and (2.3), we obtain the limit systems

$$
\left\{\begin{array}{l}
u_{t}^{0}+\phi_{x}^{0}+U_{0} u_{x}^{0}=0 \\
\phi_{t}^{0}+U_{0} \phi_{x}^{0}+\frac{1}{\lambda^{2}} u_{x}^{0}=0
\end{array}\right.
$$

and

$$
\left\{\begin{array}{l}
\xi_{t}^{0}+\alpha \xi_{x}^{0}=0 \\
\eta_{t}^{0}-\beta \eta_{x}^{0}=0
\end{array}\right.
$$


It is not clear to which solutions of (2.7) the solutions of $(2.1),(2.2)$ converge as $\varepsilon \rightarrow 0$. If we consider the system (2.8), then the most natural set of boundary conditions for $\xi^{0}, \eta^{0}$ is the following :

$$
\left\{\begin{array}{l}
\xi^{0}(0, t)=0, \\
\eta^{0}(L, t)=0,
\end{array} \quad t>0 .\right.
$$

In the $(u, \phi)$ variables, these conditions read :

$$
\left\{\begin{array}{l}
u^{0}(0, t)+\lambda \phi^{0}(0, t)=0, \\
u^{0}(L, t)-\lambda \phi^{0}(L, t)=0,
\end{array} \quad t>0 .\right.
$$

Indeed it is well-known and obvious that $(2.8),(2.9)$ - or equivalently $(2.7),(2.10)$ is a well-posed system. We define an energy for the system by setting :

$$
E^{0}(t)=\int_{0}^{L}\left\{\left(\xi^{0}(x, t)\right)^{2}+\left(\eta^{0}(x, t)\right)^{2}\right\} d x
$$

Evaluating the derivative of $E^{0}$ we find :

$$
\begin{aligned}
\frac{d}{d t} E^{0}(t) & =2 \int_{0}^{L}\left\{\xi_{t}^{0}(x, t) \xi^{0}(x, t)+\eta_{t}^{0}(x, t) \eta^{0}(x, t)\right\} d x \\
& =2 \int_{0}^{L}\left\{-\alpha \xi_{x}^{0}(x, t) \xi^{0}(x, t)+\beta \eta_{x}^{0}(x, t) \eta^{0}(x, t)\right\} d x \\
& =-\alpha\left(\xi^{0}(L, t)\right)^{2}-\beta\left(\eta^{0}(0, t)\right)^{2}
\end{aligned}
$$

and therefore

$$
\frac{d}{d t} E^{0}(t) \leq 0
$$

That is, the energy decreases in time. In a fully rigorous mathematical setting, (2.12) can be used to show that the system is well-posed, using the theory of linear contraction semi-groups (see eg. [5],[15] for the general context, and [10] for this specific system).

Actually, as we said, one of the most surprising aspects of this study was to find out that, when $\varepsilon$ goes to zero, the solutions of $(2.3),(2.6)$ converge to the system (2.8) supplemented with a different set of boundary conditions, namely :

$$
\left\{\begin{array}{l}
\xi^{0}(0, t)+\frac{\beta}{\alpha} \eta^{0}(0, t)=0, \\
\xi^{0}(L, t)+\eta^{0}(L, t)=0,
\end{array} \quad t>0 .\right.
$$


Equivalently, in the $(u, \phi)$ variables, the solutions of $(2.1),(2.2)$ converge, as $\varepsilon$ goes to zero, to the system (2.7) supplemented with the following boundary conditions :

$$
\left\{\begin{array}{l}
u(0, t)+\lambda^{2} U_{0} \phi(0, t)=0, \\
u(L, t)=0,
\end{array} \quad t>0 .\right.
$$

A full mathematical proof of this result is beyond the scope of this article; it will be presented in [10]. In the next subsection we want to prove the decay of energy for $(2.3),(2.6)$ and $(2.8),(2.13)$.

\subsection{Non-increasing energy for the systems.}

As we mentioned previously, the system (2.8) with the natural boundary condition (2.9) is well-posed. In the following, we want to give an indication of well-posedness for both $(2.3),(2.6)$ and the new limit system $(2.8),(2.13)$ by showing that some energy function is non-increasing (cf. [4]).

The system (2.8), with the boundary conditions (2.13), has a time-decreasing energy.

As in (2.11), let us define an energy for the system (2.8) :

$$
E^{0}(t)=\int_{0}^{L}\left\{\left(\xi^{0}(x, t)\right)^{2}+\left(\eta^{0}(x, t)\right)^{2}\right\} d x
$$

Then,

$$
\begin{aligned}
\frac{d}{d t} E^{0}(t) & =2 \int_{0}^{L}\left\{\xi_{t}^{0}(x, t) \xi^{0}(x, t)+\eta_{t}^{0}(x, t) \eta^{0}(x, t)\right\} d x \\
& =-\alpha\left(\left(\xi^{0}(L, t)\right)^{2}-\left(\xi^{0}(0, t)\right)^{2}\right)+\beta\left(\left(\eta^{0}(L, t)\right)^{2}-\left(\eta^{0}(0, t)\right)^{2}\right) .
\end{aligned}
$$

We now use the boundary conditions (2.13) to conclude that :

$$
\frac{d}{d t} E^{0}(t)=(\beta-\alpha)\left(\eta^{0}(L, t)\right)^{2}+\frac{\beta}{\alpha}(\beta-\alpha)\left(\left(\eta^{0}(0, t)\right)^{2} .\right.
$$

Taking into account that $\alpha>\beta$ (see (2.4)), we find that indeed $d E^{0}(t) / d t \leq 0$.

In the same way, let us prove the following :

The system (2.1), with the boundary conditions (2.2), or equivalently (2.3) with (2.6), has a time-decreasing energy. 


$$
E(t)=\int_{0}^{L}\left\{u^{2}(x, t)+\lambda^{2} \phi^{2}(x, t)\right\} d x
$$

Indeed, we have

$$
\begin{aligned}
\frac{d}{d t} E(t)= & 2 \int_{0}^{L}\left\{u_{t}(x, t) u(x, t)+\lambda^{2} \phi_{t}(x, t) \phi(x, t)\right\} d x \\
= & 2 \int_{0}^{L}\left\{u(x, t)\left(-\phi_{x}+2 \varepsilon u_{x x}+U_{0} u_{x}\right)(x, t)\right. \\
& \left.+\phi(x, t)\left(-\lambda^{2} U_{0} \phi_{x}-u_{x}\right)(x, t)\right\} d x .
\end{aligned}
$$

Using the boundary conditions, we obtain :

$$
\begin{aligned}
\frac{d}{d t} E(t) & =-2[u(x, t) \phi(x, t)]_{x=0}^{x=L}+4 \varepsilon \int_{0}^{L} u(x, t) u_{x x}(x, t) d x-\lambda^{2} U_{0} \phi^{2}(L, t) \\
& =-4 \varepsilon \int_{0}^{L} u_{x}^{2} d x-\lambda^{2} U_{0} \phi^{2}(L, t) .
\end{aligned}
$$

Finally,

$$
\frac{d}{d t} E(t) \leq 0
$$

This achieves the proof of non-increasing energy for the system (2.1)-(2.3).

Remark 2.1. : In the same way, we could have obtained the same result in the $(\xi, \eta)$ variables, defining $E(t)=\int_{0}^{L}\left\{\xi^{2}(x, t)+\eta^{2}(x, t)\right\} d x$, which is in fact identical to $(2.15)$.

Remark 2.2. : In the nonhomogeneous case and also if $F_{u}$ and $F_{\phi}$ do not vanish, the similar result can be proved. However we do not obtain then a decay of energy but a bound on the energy involving the forcing terms $F_{u}, F_{\phi}$, and the boundary values $u_{g l}, u_{g r}, \phi_{g l}$. We first extend the boundary data in the form $u_{g}(x, t), \phi_{g}(x, t)$ with :

$$
\left\{\begin{array}{l}
u_{g}(0, t)=u_{g l}(t), u_{g}(L, t)=u_{g r}(t), \quad t>0 . \\
\phi_{g}(L, t)=\phi_{g l}(t),
\end{array}\right.
$$

Then, as in [12], we set $u=u_{g}+u^{\prime}, \phi=\phi_{g}+\phi^{\prime}$, and we proceed as above, obtaining :

$$
\frac{d}{d t} E^{\prime}(t) \leq E^{\prime}(t)+\int_{0}^{L}\left(F_{u}^{\prime 2}(x, t)+\lambda^{2} F_{\phi}^{\prime 2}(x, t)\right) d x
$$


with :

$$
\left\{\begin{array}{l}
E^{\prime}(t)=\int_{0}^{L}\left(u^{\prime 2}(x, t)+\lambda \phi^{\prime 2}(x, t)\right) d x \\
F_{u}^{\prime}=F_{u}-u_{g t}-\phi_{g x}+2 \varepsilon u_{g x x}-U_{0} u_{g x}, \\
F_{\phi}^{\prime}=F_{\phi}-\phi_{g t}-U_{0} \phi_{g x}-\frac{1}{\lambda^{2}} u_{g x} .
\end{array}\right.
$$

The bound on $E^{\prime}$ follows from (2.18) and Gronwall's lemma. Note that the bound is not uniform in time in this case, unlike (2.16).

\subsection{Numerical scheme.}

Another task of this article is to propose an efficient numerical scheme for computing the solutions of system $(2.3),(2.6)$. Actually one can see, comparing the boundary conditions (2.6) with (2.14), that when $\varepsilon$ goes to zero a boundary layer shall appear at $x=0$. The approximation of such a problem by a classical discrete method (finite elements or finite differences) would require the use of a fine mesh, at least of the order of the size of the boundary layer, that is here $\mathcal{O}(\varepsilon)$. To avoid using such a small and expensive mesh, it was proposed in [2], [3] for a similar problem (with one equation only), to add to the Galerkin basis a function representing the boundary layer corrector in the context of a finite element discretization ${ }^{1}$.

In spite of the time dependence of the equations, we want to focus in this article on the space discretization, taking into account that the time does not change the existence of the boundary layers.

In a classical finite element method we consider the standard piecewise linear base functions

$$
\varphi_{j}(x)=\left\{\begin{array}{ll}
\frac{x-x_{j-1}}{h} & \text { for } x_{j-1} \leq x \leq x_{j}, \\
\frac{x_{j+1}-x}{h} & \text { for } x_{j} \leq x \leq x_{j+1}, \\
0 & \text { elsewhere, }
\end{array} \quad 1 \leq j \leq M-1 .\right.
$$

$$
\varphi_{M}(x)= \begin{cases}\frac{x-x_{M-1}}{h} & \begin{array}{l}
\text { for } x_{M-1} \leq x \leq x_{M} \\
\text { elsewhere }
\end{array}\end{cases}
$$

where $h=L / M, x_{j}=j h$, and we look for an approximate solution

\footnotetext{
${ }^{1}$ This assumes of course that the shape of the corrector is known, see Remark 2.3. The case of finite differences will be discussed in a forthcoming article.
} 


$$
\left(\begin{array}{c}
\xi_{M} \\
\eta_{M}
\end{array}\right) \in\left(\begin{array}{c}
\xi_{g} \\
\eta_{g}
\end{array}\right)+V_{M}
$$

where $\xi_{g}=u_{g}+\lambda \phi_{g}, \eta_{g}=u_{g}-\lambda \phi_{g}$, and

$$
V_{M}=\operatorname{Span}\left\{\left(\begin{array}{c}
\varphi_{1} \\
0
\end{array}\right),\left(\begin{array}{c}
0 \\
\varphi_{1}
\end{array}\right), \cdots,\left(\begin{array}{c}
\varphi_{M-1} \\
0
\end{array}\right),\left(\begin{array}{c}
0 \\
\varphi_{M-1}
\end{array}\right),\left(\begin{array}{c}
\varphi_{M} \\
-\varphi_{M}
\end{array}\right)\right\}
$$

The approximate solution satisfies :

$(2.23)$

$$
\left\{\begin{array}{l}
\frac{d}{d t}\left(\xi_{M}, \chi_{1}\right)+\alpha\left(\xi_{M_{x}}, \chi_{1}\right)+\varepsilon\left(\xi_{M_{x}}+\eta_{M_{x}}, \chi_{1_{x}}\right)=0, \\
\frac{d}{d t}\left(\eta_{M}, \chi_{2}\right)-\beta\left(\eta_{M_{x}}, \chi_{2}\right)+\varepsilon\left(\xi_{M_{x}}+\eta_{M_{x}}, \chi_{2_{x}}\right)=0,
\end{array} \quad \forall \chi=\left(\begin{array}{c}
\chi_{1} \\
\chi_{2}
\end{array}\right) \in V_{M},\right.
$$

where $(f, g)=\int_{0}^{L} f(x) g(x) d x$ is the usual $\mathbb{L}^{2}$ inner product. A similar weak formulation of $(2.3)$ is also valid.

Unfortunately, the numerical results are not satisfying as soon as $h \gtrsim \varepsilon$. To improve the numerical resolution, we add the following corrector :

$$
\tilde{\varphi}_{0}(x)=e^{-r x / \varepsilon} \text {, where } r=\frac{\alpha \beta}{\alpha-\beta}>0 .
$$

To insert this corrector in the basis functions, and make it consistent with the boundary conditions, we slightly modify $\tilde{\varphi}_{0}$ and replace it with :

$$
\varphi_{0}(x)=e^{-\frac{r x}{\varepsilon}}+\left(1-e^{-\frac{r L}{\varepsilon}}\right) \frac{x}{L}-1
$$

The expression of $\varphi_{0}$ is very similar to what appears in [2] and [3], but since we have two coupled equations the corrector here is more difficult to determine.

The space $V_{M}$ is replaced by :

$$
\tilde{V}_{M}=\operatorname{Span}\left\{\left(\begin{array}{c}
\varphi_{0} \\
0
\end{array}\right),\left(\begin{array}{c}
0 \\
\varphi_{0}
\end{array}\right), \ldots,\left(\begin{array}{c}
\varphi_{M-1} \\
0
\end{array}\right),\left(\begin{array}{c}
0 \\
\varphi_{M-1}
\end{array}\right),\left(\begin{array}{c}
\varphi_{M} \\
-\varphi_{M}
\end{array}\right)\right\}
$$

The new formulation reads :

$$
\left(\begin{array}{c}
\xi_{M} \\
\eta_{M}
\end{array}\right) \in\left(\begin{array}{c}
\xi_{g} \\
\eta_{g}
\end{array}\right)+\tilde{V}_{M}, \text { and : }
$$


$(2.26)$

$\left\{\begin{array}{l}\frac{d}{d t}\left(\xi_{M}, \chi_{1}\right)+\alpha\left(\xi_{M_{x}}, \chi_{1}\right)+\varepsilon\left(\xi_{M_{x}}+\eta_{M_{x}}, \chi_{1_{x}}\right)=0, \\ \frac{d}{d t}\left(\eta_{M}, \chi_{2}\right)-\beta\left(\eta_{M_{x}}, \chi_{2}\right)+\varepsilon\left(\xi_{M_{x}}+\eta_{M_{x}}, \chi_{2_{x}}\right)=0,\end{array} \quad \forall \xi=\left(\begin{array}{c}\chi_{1} \\ \chi_{2}\end{array}\right) \in \tilde{V}_{M}\right.$.

One can easily observe that (2.26) is a linear differential system for the coefficients $a_{j}, b_{j}$ defining $\xi_{M}$ and $\eta_{M}$ :

$$
\left\{\begin{array}{l}
\xi_{M}(x, t)=\xi_{g}(x, t)+a_{0}(t) \varphi_{0}(x)+\sum_{j=1}^{M} a_{j}(t) \varphi_{j}(x), \\
\eta_{M}(x, t)=\eta_{g}(x, t)+b_{0}(t) \varphi_{0}(x)+\sum_{j=1}^{M} b_{j}(t) \varphi_{j}(x) .
\end{array}\right.
$$

Remark 2.3. : The corrector $\varphi_{0}$ was not known at the beginning of our work. To perform the numerical simulations which led us to the boundary conditions (2.13), we used an empirical parabolic corrector $\varphi_{0}^{*}$ which was used in the algorithm above instead of $\varphi_{0}$. Further applications of this empirical corrector will be discussed elsewhere.

\subsection{Numerical results.}

We emphasize the case where $u_{g}, v_{g}, \phi_{g}$ vanish which leads to more intuitive figures. The case where these data do not vanish do not raise any additional numerical difficulty : an example is given at the end of the section.

The results are presented below in the $(\xi, \eta)$ variables, because the plots are more readable. We will use the same initial data for $\xi$ and $\eta$, corresponding to the bump plotted in Figure 1. For the computations shown hereafter, we use these values of the parameters :

$$
\begin{gathered}
\left\{\begin{array}{l}
U_{0}=1, \\
\lambda=1 / 2,
\end{array}\right. \\
\left\{\begin{array}{l}
\varepsilon=10^{-3} \\
M=
\end{array} \quad \begin{array}{l}
\alpha=3 \\
\beta=1 .
\end{array}\right.
\end{gathered}
$$

For $t=0.15$, for either the classical finite element method or the new algorithm proposed, we have the same numerical results (see Figure 2) : $\xi$ is travelling to the right with a speed $\alpha$, whereas $\eta$ is moving to the left with a speed $\beta$. On Figure $2, \xi$ is going out of the frame at the boundary $x=L$, and $\eta$ is coming in. This illustrates the boundary condition $\xi+\eta=0$. We can also notice a decrease of the $\mathbb{L}^{\infty}$-norm, due to the diffusion $\varepsilon$. 
As soon as the boundary $x=0$ is reached (here at $t=0.45$ ), the classical method is no longer satisfying and the boundary layer can not be computed with such a small number of points $\left(\varepsilon=10^{-3}, M=200\right)$ : see Figure 3, displaying a zoom at $x=0$. On the contrary, with the same values of the parameters, the method using the new function $\varphi_{0}$ gives very good results, even if $h>\varepsilon$ (Figure 4).

We now want to show the plots that confirm the convergence of $(2.3),(2.6)$ to (2.8),(2.13). To this end we compare the two systems at $t=0.5$ for different values of the parameter $\varepsilon$ ( $\alpha$ and $\beta$ are unchanged). Figure 5 presents the numerical solution of the limit system $(\varepsilon=0)$. On can notice that the values of $\xi^{0}$ and $\eta^{0}$ satisfy the boundary conditions (2.13). Figures 6 and 7 respectively display the numerical solutions of (2.3) with $\varepsilon=10^{-2}$ and $\varepsilon=10^{-5}$. They illustrate the convergence indicated. Figure 8 displays a zoom of $(\xi, \eta)$ at the boundary $x=0$ for $\varepsilon=10^{-5}$, in order to see the boundary layer that has just appeared.

To finish with this section, we show two plots with nonhomogeneous boundary conditions. The two following figures show the numerical solutions of the system for $\xi_{g l}=-0.05, \eta_{g l}=0.1$, and $u_{g r}=0.1$. Figure 9 is the initial data, and Figure 10 plots the solutions at $t=0.65$, with $\varepsilon=10^{-6}$. Some other tests, with boundary conditions depending on time have also been done, but since the difficulty is not here we chose not to show them.

\section{TRANSPARENT BOUNDARY CONDITIONS}

The previous numerical results show that the limit system is not what one could believe at first. Despite the mathematical interest of this study, the reflections that appeared at the boundaries may not be physically relevant, and this is why we consider hereafter a set of transparent boundary conditions (TBC) removing these reflections. They enjoy the following two important properties :

- for $\varepsilon>0$ there is no reflection at the boundaries.

- the boundary conditions for the limit system $(\varepsilon \rightarrow 0)$ are the natural conditions (2.10), identical to $(2.9)$.

The aim of the following is to provide numerical evidence of these properties of the TBC. The reader is referred to [10] for an alternate set of transparent conditions with full mathematical proofs of existence and uniqueness of solutions and convergence as $\varepsilon \rightarrow 0$.

\subsection{The boundary conditions.}

We are now interested in studying the same system (2.3), but with a set of boundary conditions that would avoid the boundary layers and reflections which appeared 
in the previous section.

For system (2.3), we will use the following transparent boundary conditions which are consistant with (2.8) :

$$
\left\{\begin{array}{l}
\xi(0, t)=\xi_{g l}(t) \\
\eta(L, t)=\eta_{g r}(t) \\
\eta_{t}(0, t)-\beta\left(\xi_{x}+\eta_{x}\right)(0, t)=f_{\eta}(0, t)-\frac{\beta}{\alpha} f_{\xi}(0, t), \\
\xi_{t}(L, t)+\alpha\left(\xi_{x}+\eta_{x}\right)(L, t)=f_{\xi}(L, t)-\frac{\alpha}{\beta} f_{\eta}(L, t),
\end{array} \quad t>0,\right.
$$

where $\xi_{g l}$ and $\eta_{g r}$ are given.

The system consisting of (2.3) and (3.1) is obviously equivalent to (2.1) supplemented with the following boundary conditions :

$$
\left\{\begin{array}{l}
u(0, t)+\lambda \phi(0, t)=\xi_{g l}(t), \\
u(L, t)-\lambda \phi(L, t)=\eta_{g r}(t), \\
u_{t}(0, t)-\lambda \phi_{t}(0, t)-2 \beta u_{x}(0, t)=f_{\eta}(0, t)-\frac{\beta}{\alpha} f_{\xi}(0, t), \quad t>0 . \\
u_{t}(L, t)+\lambda \phi_{t}(L, t)+2 \alpha u_{x}(L, t)=f_{\xi}(L, t)-\frac{\alpha}{\beta} f_{\eta}(L, t),
\end{array}\right.
$$

Here, even when the parameter $\varepsilon$ tends to zero, there is no boundary layer for the functions themselves, neither at $x=0$ nor at $x=L$. On the contrary to the previous section, we do not need to improve the numerical algorithm because of the absence of boundary layer. Therefore we can choose $h$ greater than $\varepsilon$ with a classical finite element method, and the motion looks very regular, even close to the boundary.

We first check that the proposed TBC lead to a decreasing energy for the system, and then we show numerically the convergence of $(2.1),(3.2)$ to $(2.7),(2.10)$ - or equivalently of $(2.3),(3.1)$ to $(2.8),(2.9)$ - when $\varepsilon$ goes to zero.

\subsection{Non-increasing energy for the system.}

As we already did in Section 2.2 for system (2.3) with boundary conditions (2.6), we want to prove that the energy of the system $(2.1),(3.2)$ does not increase with time in the absence of forcing, that is :

$$
\xi_{g l}=\eta_{g r}=0 .
$$

The system (2.1), with the boundary conditions (3.2), or equivalently (2.3) with (3.1), and (3.3), has a time-decreasing energy. 
As in (2.11), let us define an energy for the system (2.1) :

$$
E(t)=\int_{0}^{L}\left\{u^{2}(x, t)+\lambda^{2} \phi^{2}(x, t)\right\} d x,
$$

and let us evaluate the derivative :

$$
\begin{aligned}
\frac{d}{d t} E(t)= & 2 \int_{0}^{L}\left\{u_{t}(x, t) u(x, t)+\lambda^{2} \phi_{t}(x, t) \phi(x, t)\right\} d x \\
= & 2 \int_{0}^{L}\left\{u(x, t)\left(-\phi_{x}+2 \varepsilon u_{x x}-U_{0} u_{x}\right)(x, t)+\phi(x, t)\left(-\lambda^{2} U_{0} \phi_{x}-u_{x}\right)(x, t)\right\} d x \\
= & -2[u(x, t) \phi(x, t)]_{x=0}^{x=L}+4 \varepsilon \int_{0}^{L} u(x, t) u_{x x}(x, t) d x \\
& +U_{0}\left(u^{2}(0, t)+\lambda \phi^{2}(0, t)-u^{2}(L, t)-\lambda \phi^{2}(L, t)\right)
\end{aligned}
$$

Using the boundary conditions (3.2a),(3.2c), and recalling that $\alpha=U_{0}+1 / \lambda$, $\beta=-U_{0}+1 / \lambda$, we obtain :

$$
\begin{aligned}
\frac{d}{d t} E(t)= & -2 \alpha u^{2}(L, t)-2 \beta u^{2}(0, T)-4 \varepsilon \int_{0}^{L} u_{x}^{2}(x, t) d x \\
& +4 \varepsilon\left[u(x, t) u_{x}(x, t)\right]_{x=0}^{x=L}
\end{aligned}
$$

Taking into account $(3.2 \mathrm{~b}),(3.2 \mathrm{~d})$, we evaluate the following term :

$$
\begin{aligned}
4 \varepsilon\left[u(x, t) u_{x}(x, t)\right]_{x=0}^{x=L}= & -\frac{2 \varepsilon}{\alpha} u(L, t)\left(u_{t}(L, t)+\lambda \phi_{t}(L, t)\right) \\
& -\frac{2 \varepsilon}{\beta} u(0, t)\left(u_{t}(0, t)-\lambda \phi_{t}(L, t)\right) \\
= & -2 \varepsilon \frac{d}{d t}\left(\frac{u^{2}(L, t)}{\alpha}+\frac{u^{2}(0, t)}{\beta}\right)
\end{aligned}
$$

From (3.5),(3.6), and since $\alpha, \beta>0$, we find :

$$
\frac{d}{d t}\left\{E(t)+\frac{2 \varepsilon}{\alpha} u^{2}(L, t)+\frac{2 \varepsilon}{\beta} u^{2}(0, t)\right\} \leq 0 .
$$

Hence,

$$
\begin{aligned}
E(t)+\frac{2 \varepsilon}{\alpha} u^{2}(L, t)+\frac{2 \varepsilon}{\beta} u^{2}(0, t) & \leq E(0)+\frac{2 \varepsilon}{\alpha} u^{2}(L, 0)+\frac{2 \varepsilon}{\beta} u^{2}(0,0) \\
& =E(0)
\end{aligned}
$$

Finally :

$$
E(t) \leq E(0)
$$


This achieves the proof of non-increasing energy for the system (2.1) with boundary conditions (3.2).

Remark 3.1. : We could have obtained a similar result in the $(\xi, \eta)$ variables, defining $E(t)=\int_{0}^{L}\left\{\xi^{2}(x, t)+\eta^{2}(x, t)\right\} d x$, which is identical to (3.4).

Remark 3.2. : In the presence of forcing, i.e. if $F_{u}, F_{\phi}, \xi_{g l}, \eta_{g r}$ do not vanish, we proceed as in Remark 2.2.

\subsection{Numerical Scheme.}

As there is no boundary layer to compute, we can implement the classical finite element method. We do not need to use $\varphi_{0}$ anymore, but we will replace it with the classical $\varphi_{0}$ (see below) as the functions do not verify $\xi(0, t)=\eta(0, t)=0$ anymore. Then, as we already did in Section 2, we extend the boundary data inside the interval $(0, L)$ in the form $\xi_{g}(x, t), \eta_{g}(x, t)$ with :

$$
\left\{\begin{array}{l}
\xi_{g}(0, t)=\xi_{g l}(t), \\
\eta_{g}(L, t)=\eta_{g r}(t),
\end{array} \quad t>0 .\right.
$$

Hence we will look for an approximate function :

$$
\left(\begin{array}{c}
\xi_{M} \\
\eta_{M}
\end{array}\right) \in\left(\begin{array}{c}
\xi_{g} \\
\eta_{g}
\end{array}\right)+V_{M}
$$

with :

$$
V_{M}=\operatorname{Span}\left\{\left(\begin{array}{c}
\varphi_{0} \\
0
\end{array}\right),\left(\begin{array}{c}
0 \\
\varphi_{0}
\end{array}\right), \cdots,\left(\begin{array}{c}
\varphi_{M} \\
0
\end{array}\right),\left(\begin{array}{c}
0 \\
\varphi_{M}
\end{array}\right)\right\}
$$

where $\varphi_{j}, 1 \leq j \leq M$ are as in (2.20),(2.21), and :

$$
\varphi_{0}(x)= \begin{cases}\frac{x_{1}-x}{h} & \text { for } 0 \leq x \leq x_{1}, \\ 0 & \text { elsewhere. }\end{cases}
$$

The approximate solution $\left(\xi_{M}, \eta_{M}\right)$ satisfies :

$$
\left\{\begin{array}{l}
\xi_{M}(x, t)=\xi_{g}(t)+\sum_{j=0}^{M} a_{j}(t) \varphi_{j}(x), \\
\eta_{M}(x, t)=\eta_{g}(t)+\sum_{j=0}^{M} b_{j}(t) \varphi_{j}(x) .
\end{array}\right.
$$

One more time, there is a linear differential system to solve to determine the coefficients $\left(a_{j}, b_{j}\right)$, which is then solved by time discretization. This is all classical and 
no more details are needed.

\subsection{Numerical results.}

Again, we will show some plots with the variables $(\xi, \eta)$. Also, as we did in Section 2 , the results are mainly presented with homogeneous boundary conditions. An example of nonhomogeneous case is given in the last figures.

The aim of the following is to show that the system (2.3), with the boundary conditions (3.1), converges to the equations (2.8),(2.9) when $\varepsilon$ goes to zero.

The decoupled system (2.8),(2.9) consists of two transport equations and the exact solutions are the following functions :

$$
\left\{\begin{array}{l}
\xi^{0}(x, t)=\xi_{0}(x-\alpha t), \\
\eta^{0}(x, t)=\eta_{0}(x+\beta t) .
\end{array}\right.
$$

The following plots illustrate the convergence announced. Figure 11 represents the initial functions $\xi_{0}=\eta_{0}$, same as those in Figure 1. Figures 12, 13 and 14 represent $\xi$ and $\eta$ at time $t=0.15$, for different values of $\varepsilon$ (respectively $10^{-3}, 10^{-4}$ and $\left.10^{-5}\right)$. For these 3 plots, we can notice that there is no reflection at $x=L$. Figure 14 really looks like the exact solution (3.13), at the precision of the figure.

In Figure 15, we also set $\varepsilon=10^{-5}$, but $t=0.45 ; \xi$ now equals to zero everywhere, and $\eta$ is getting out through the boundary $x=0$. We can notice that there is naturally no boundary layer here. Finally, Figure 16 shows the numerical solutions after a time (here $t=1$ ) such that both of them equal zero.

As we did for the Dirichlet boundary conditions, we end this section with a nonhomogeneous case, namely with $\xi_{g l}=0.05, \eta_{g r}=-.1$. Figure 17 shows the initial condition, whereas Figure 18 gives the solution at time $t=0.3$.

\section{Conclusion}

In this article, a linear differential system consisting of two coupled scalar evolution equations in space dimension one was considered ; this system was derived from a modal analysis of the Primitive Equations of the ocean. We have shown numerically that, by adjunction of a small viscosity, the system converges to some unusual, unexpected limit system thus producing boundary layers and reflections of waves at the boundary. We have also proposed an alternate set of boundary conditions of 
transparent type for the viscous systems and, in this case, the viscous system does not produce boundary layers nor reflections of waves at the boundary.

For the Primitive Equations of the atmosphere we learned from this study, that a mild friction term can produce unexpected spurious waves in the case of a limited domain. We leave to subsequent studies the choice between accepting such undesirable waves and coping with them, versus the implementation of more involved schemes.

\section{Acknowledgements.}

This work was partially supported by the National Science Foundation under the grants NSF-DMS-0074334 and NSF-DMS-0305110, and by the Research Fund of Indiana University. 


\section{REFERENCES}

[1] H. Brézis. Opérateurs maximaux monotones et semi-groupes de contractions dans les espaces de Hilbert. North-Holland Publishing Co., Amsterdam, 1973. North-Holland Mathematics Studies, No. 5. Notas de Matemática (50).

[2] W. Cheng and R. Temam. Numerical approximation of one-dimensional stationary diffusion equations with boundary layers. Comput. \& Fluids, 31(4-7):453-466, 2002.

[3] W. Cheng, R. Temam, and X. Wang. New approximation algorithms for a class of partial differential equations displaying boundary layer behavior. Methods Appl. Anal., 7(2):363-390, 2000.

[4] D. Gottlieb. Personal communication. Manuscript.

[5] J.L. Lions. Problèmes aux limites dans les équations aux dérivées partielles. Les Presses de l'Université de Montréal, Montreal, Que., 1965. Reedited in [?].

[6] J.L. Lions, R. Temam, and S.H. Wang. New formulations of the primitive equations of atmosphere and applications. Nonlinearity, 5(2):237-288, 1992.

[7] J.L. Lions, R. Temam, and S.H. Wang. On the equations of the large-scale ocean. Nonlinearity, 5(5):1007-1053, 1992.

[8] J. Oliger and A. Sundström. Theoretical and practical aspects of some initial boundary value problems in fluid dynamics. SIAM J. Appl. Math., 35(3):419-446, 1978.

[9] J. Pedlosky. Geophysical fluid dynamics, 2ed. Springer, 1987.

[10] A. Rousseau and R. Temam. Article in preparation, 2003.

[11] R. Salmon. Lectures on geophysical fluid dynamics. Oxford University Press, New York, 1998.

[12] R. Temam and J. Tribbia. Open boundary conditions for the primitive and boussinesq equations. Journ. of Atmosph. Research, to appear, 2003.

[13] R. Temam and M. Ziane. Some mathematical problems in geophysical fluid dynamics. In S. Friedlander and D. Serre, editors, Handbook of mathematical fluid dynamics. North-Holland, 2003.

[14] W. Washington and C. Parkinson. An introduction to three-dimensional climate modelling. Oxford Univ. Press, 1986.

[15] K. Yosida. Functional analysis. Springer-Verlag, Berlin, sixth edition, 1980. 
Figure Captions

FIG.1. Dirichlet Boundary Condition. Initial data $\xi_{0}, \eta_{0}$.

FIG.2. Dirichlet Boundary Condition, $\xi$ and $\eta$ at $t=0.15$.

FIG.3. Oscillating Solution without numerical corrector, with $\varepsilon=10^{-3}, t=0.45$.

FIG.4. Nonoscillating Solution with numerical corrector (same $\varepsilon$ and $t$ as in FIG.3).

FIG.5. Limit Solution, $\varepsilon=0, t=0.5$.

FIG.6. Solution with corrector, with $\varepsilon=10^{-2}, t=0.5$.

FIG.7. Solution with corrector, with $\varepsilon=10^{-5}, t=0.5$.

FIG.8. Zoom at $x=0$ of FIG.7

FIG.9. Initial data for Nonhomogeneous Dirichlet Boundary Conditions.

FIG.10. Solution with corrector, $\varepsilon=10^{-6}, t=0.65$ (nonhomogeneous boundary conditions).

FIG.11. Transparent Boundary Condition. Initial data $\xi_{0}, \eta_{0}$.

FIG.12. Solution with Transparent Boundary Condition, $\varepsilon=10^{-3}, t=0.15$.

FIG.13. Solution with Transparent Boundary Condition, $\varepsilon=10^{-4}, t=0.15$.

FIG.14. Solution with Transparent Boundary Condition, $\varepsilon=10^{-5}, t=0.15$.

FIG.15. Solution with Transparent Boundary Condition, $\varepsilon=10^{-5}, t=0.45$. The right wave has crossed the right boundary.

FIG.16. Solution with Transparent Boundary Condition, $\varepsilon=10^{-5}, t=1.0$. Both waves have left the domain.

FIG.17. Initial data for Nonhomogeneous Transparent Boundary Conditions.

FIG.18. Solution with Transparent Boundary Condition, $\varepsilon=10^{-5}, t=0.3$ (nonhomogeneous boundary conditions). 


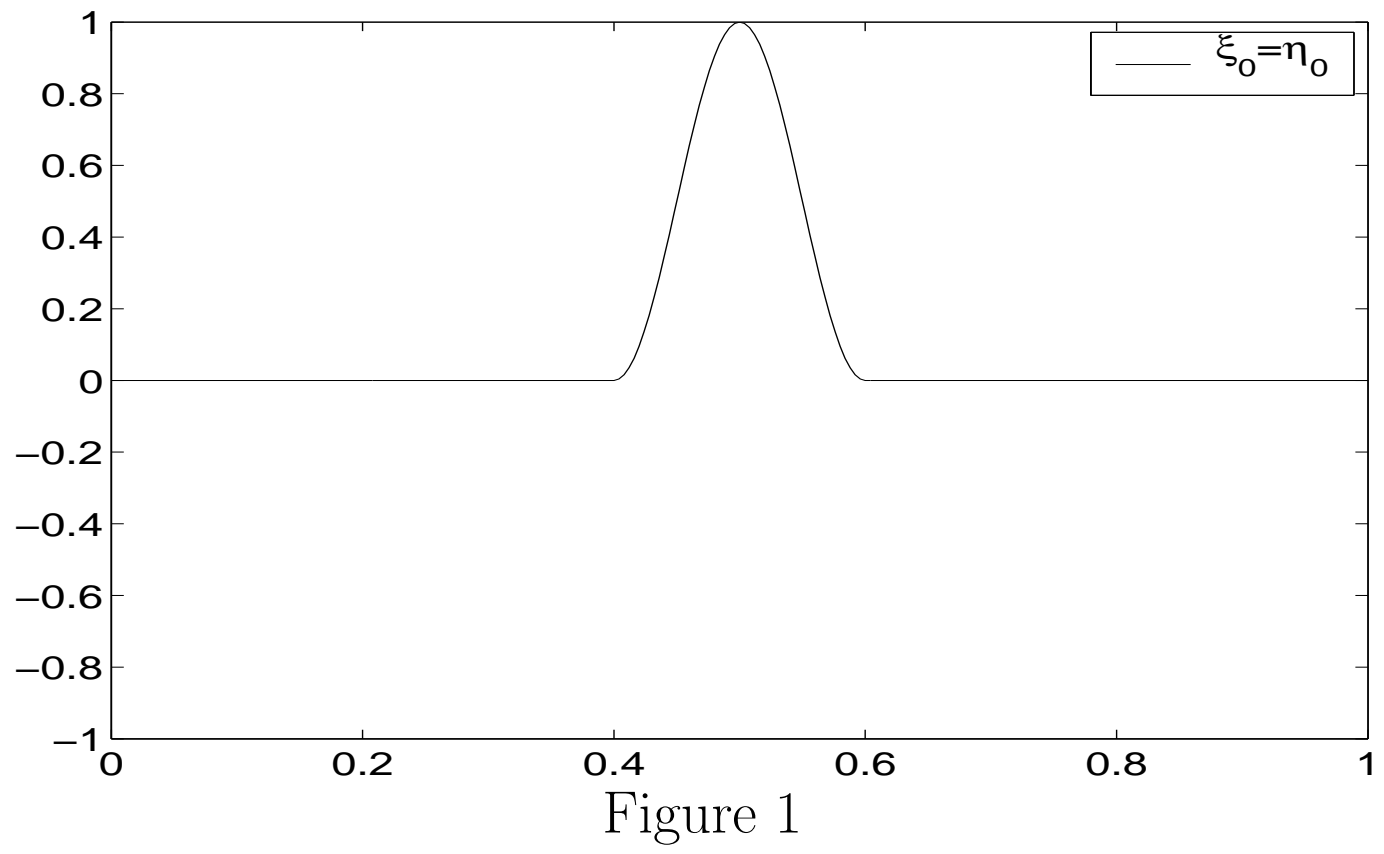


ROUSSEAU, TEMAM, AND TRIBBIA

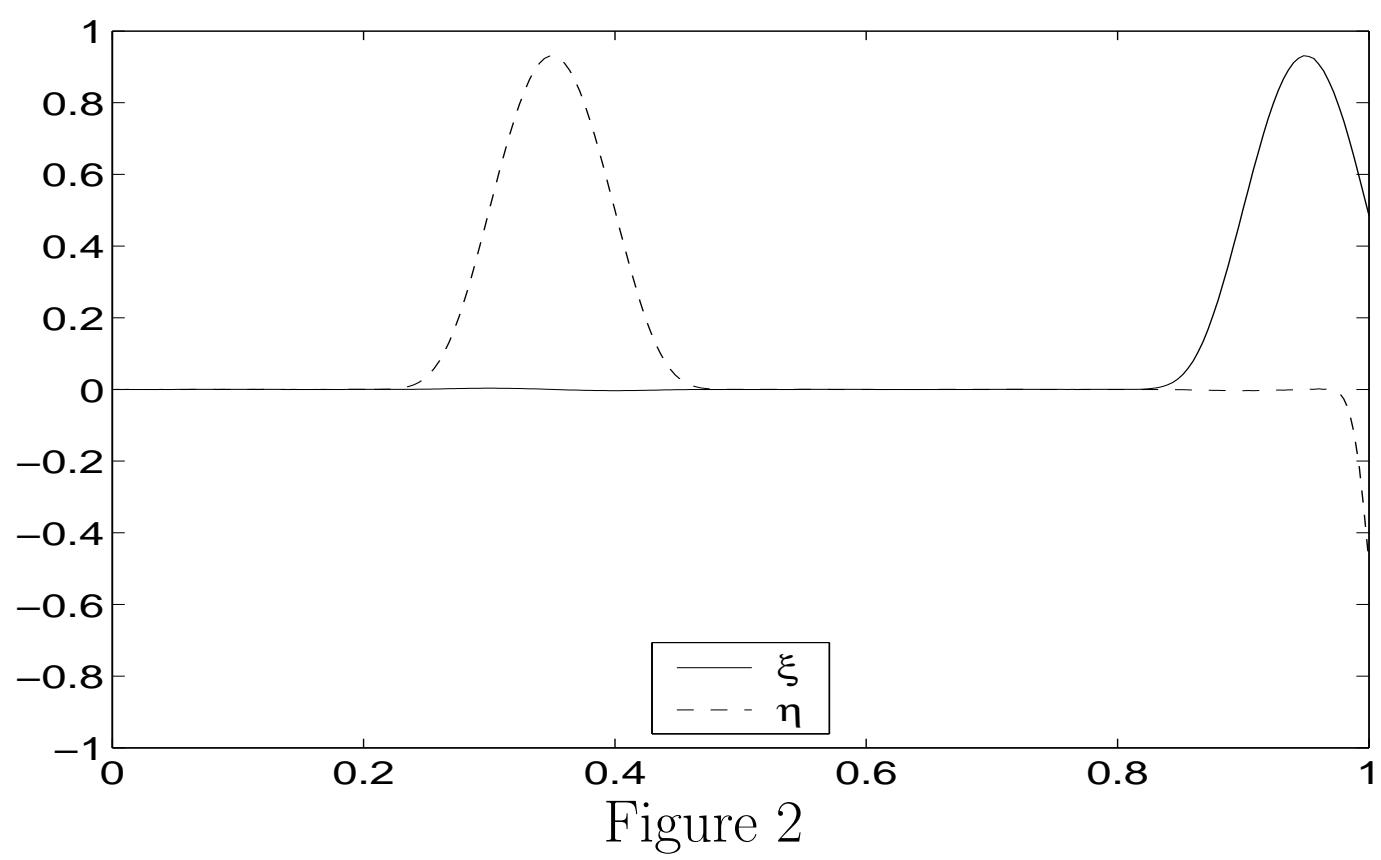




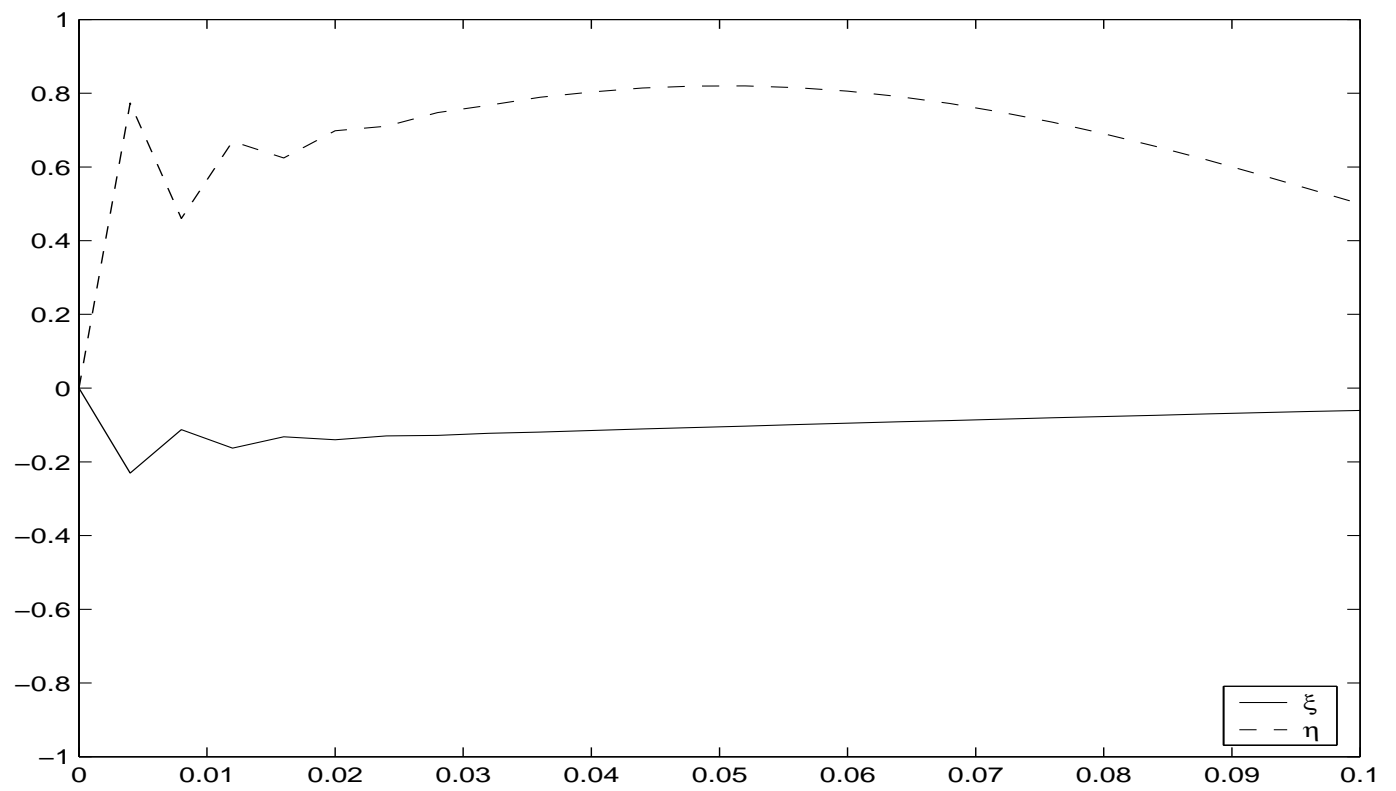

Figure 3 
ROUSSEAU, TEMAM, AND TRIBBIA

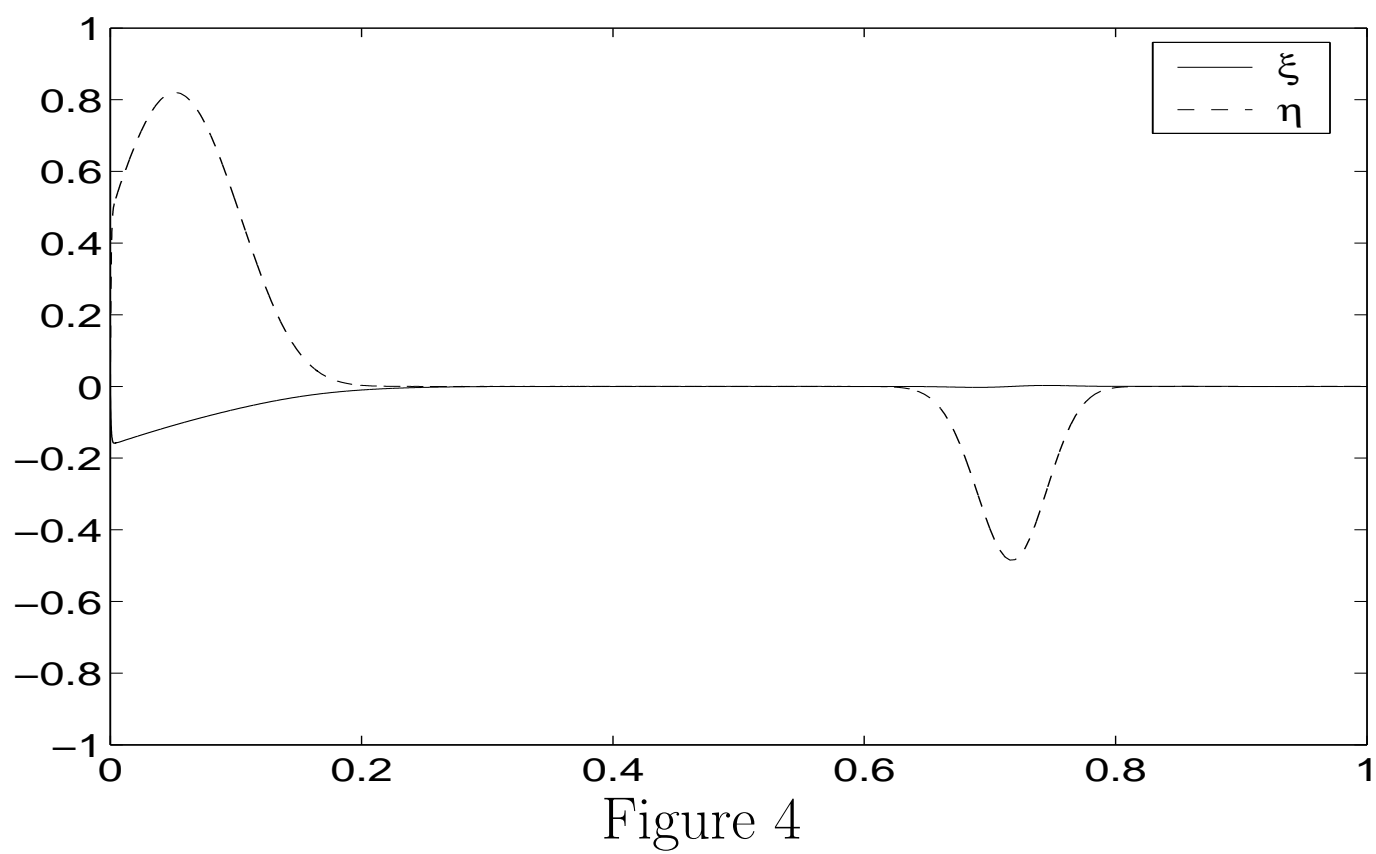


BOUNDARY LAYERS IN AN OCEAN RELATED SYSTEM

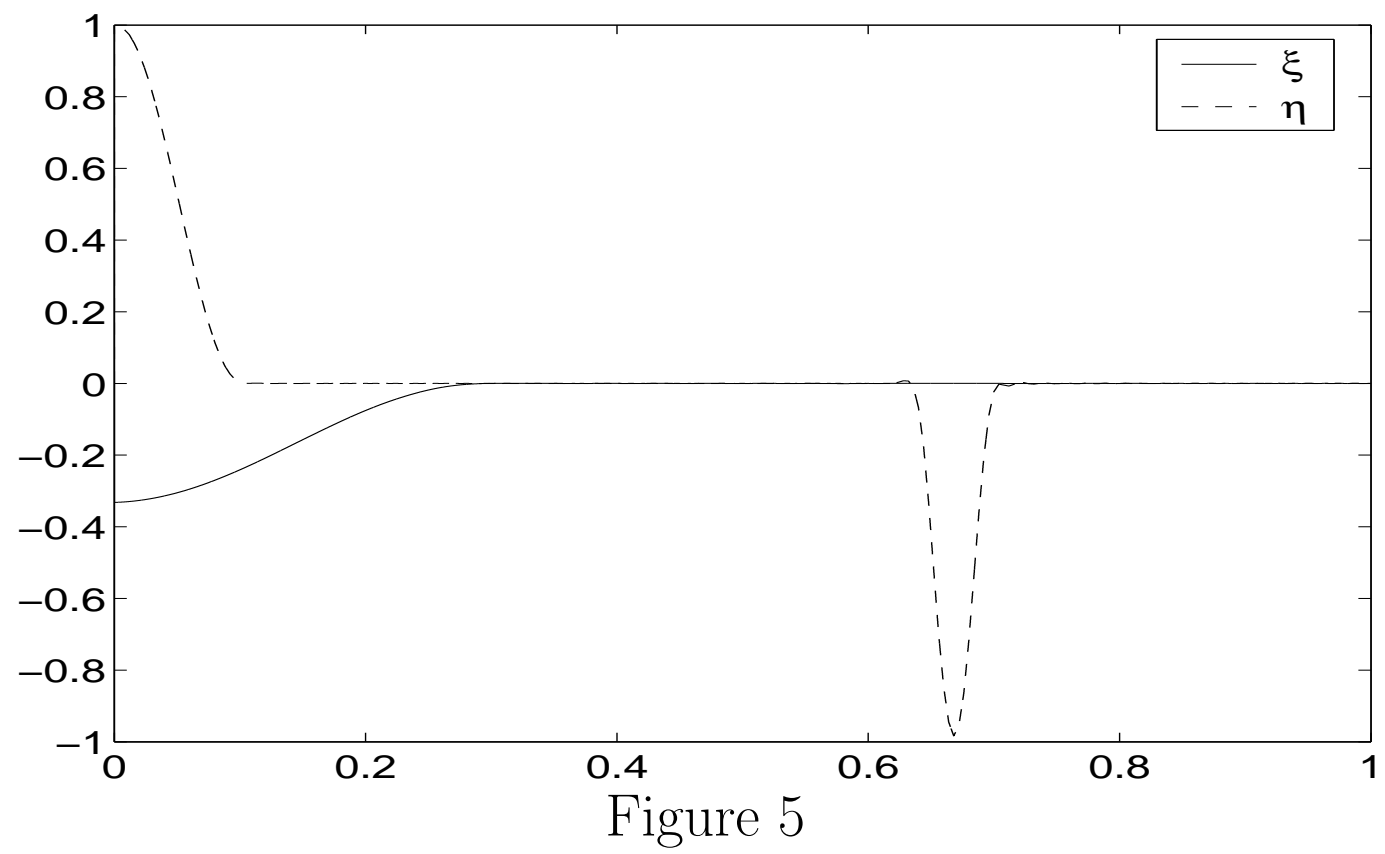


ROUSSEAU, TEMAM, AND TRIBBIA

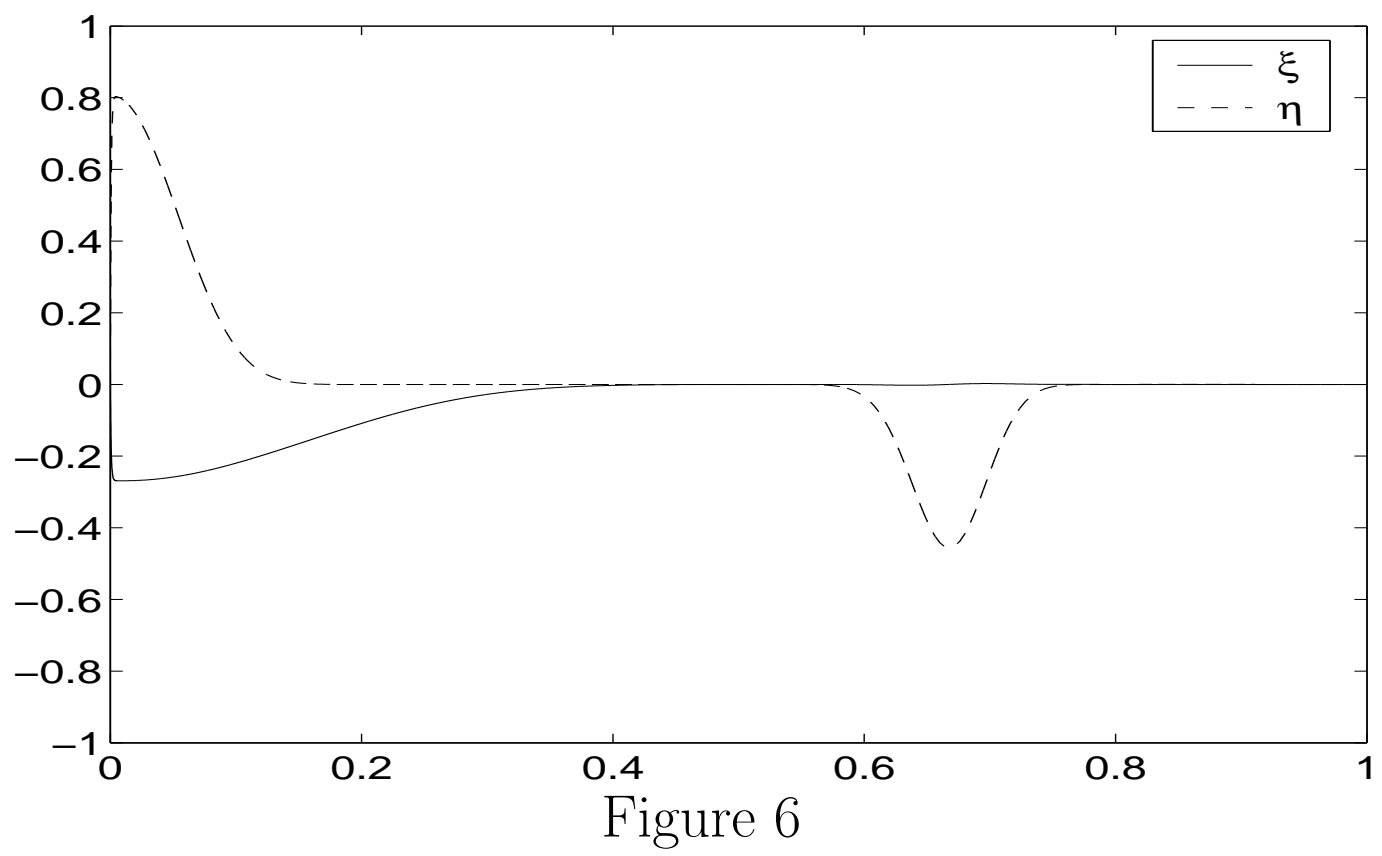


BOUNDARY LAYERS IN AN OCEAN RELATED SYSTEM

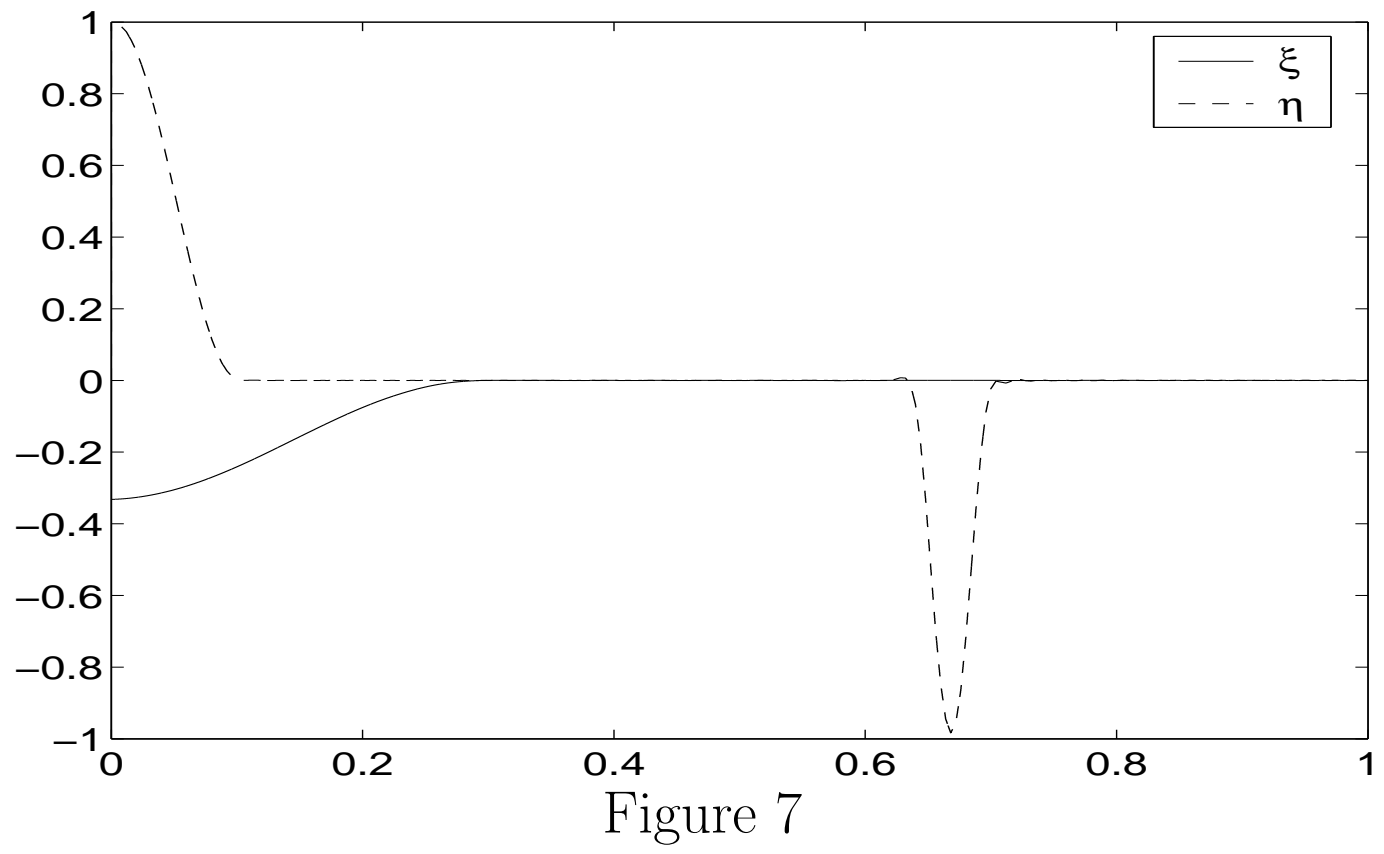


ROUSSEAU, TEMAM, AND TRIBBIA

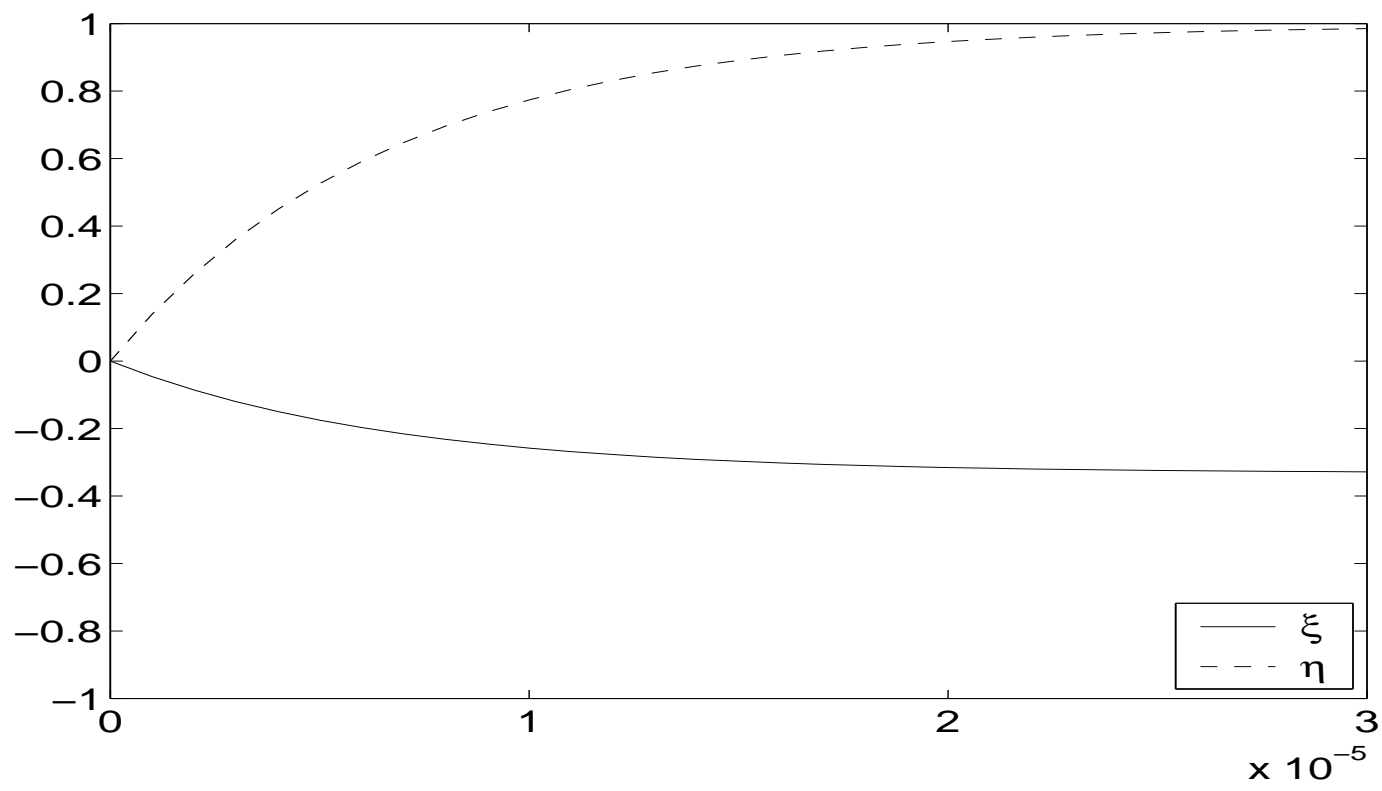

Figure 8 
BOUNDARY LAYERS IN AN OCEAN RELATED SYSTEM

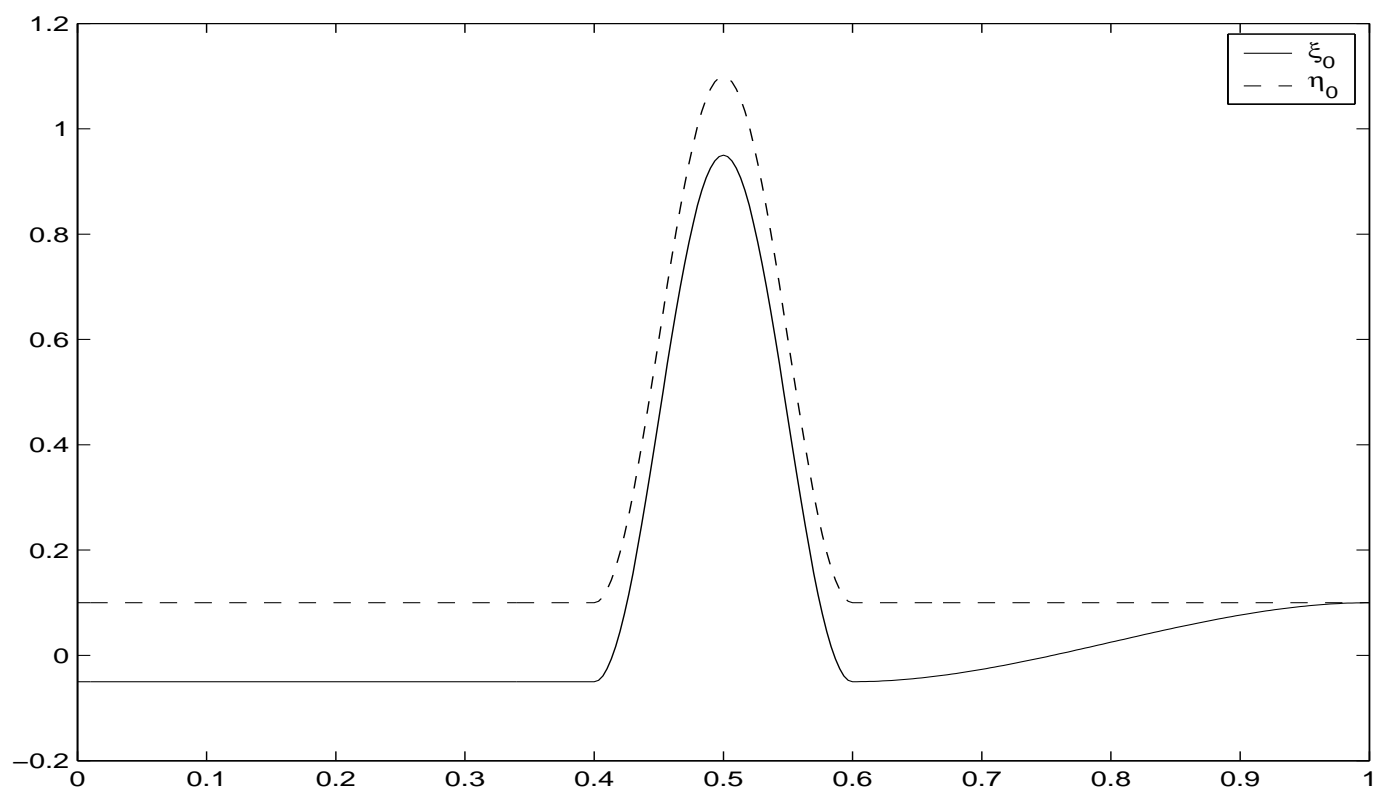

Figure 9 
ROUSSEAU, TEMAM, AND TRIBBIA

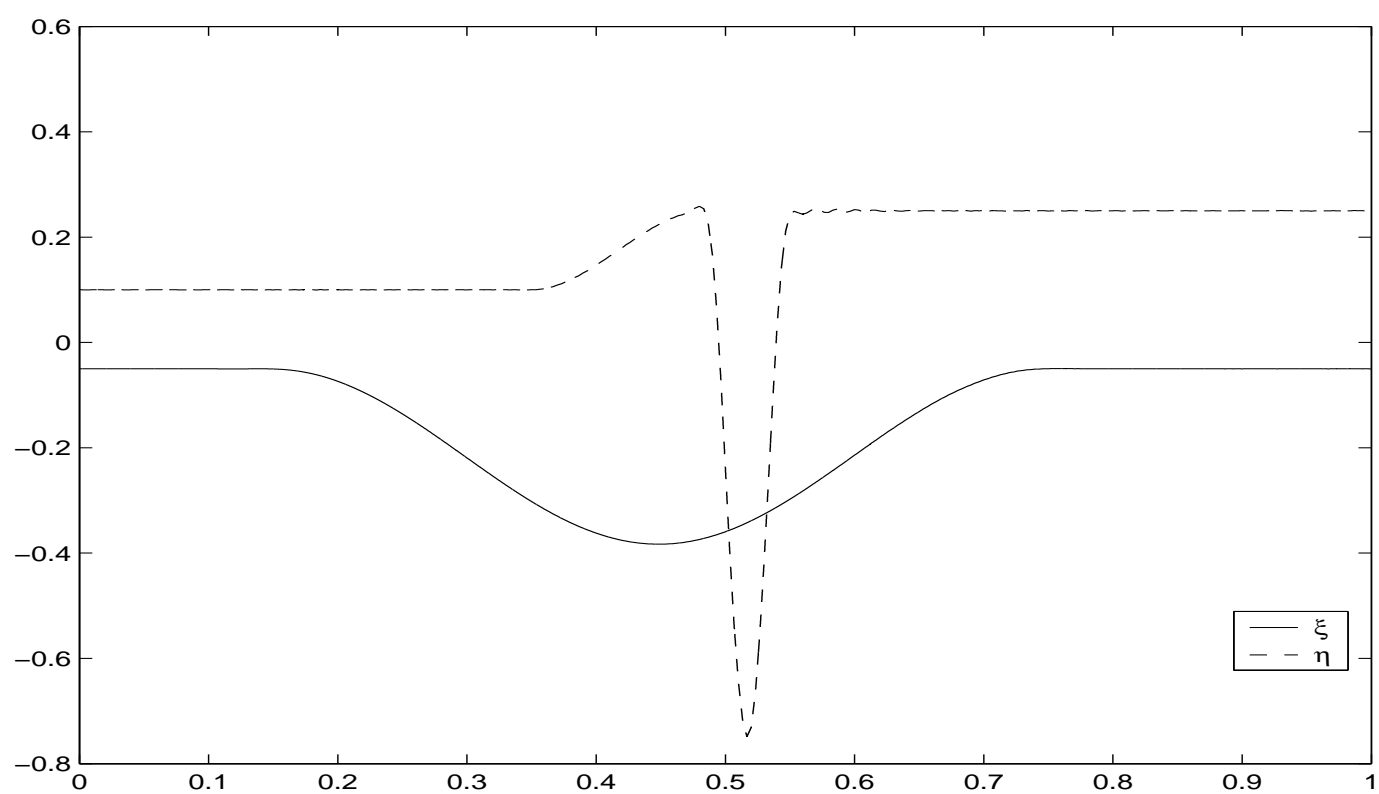

Figure 10 


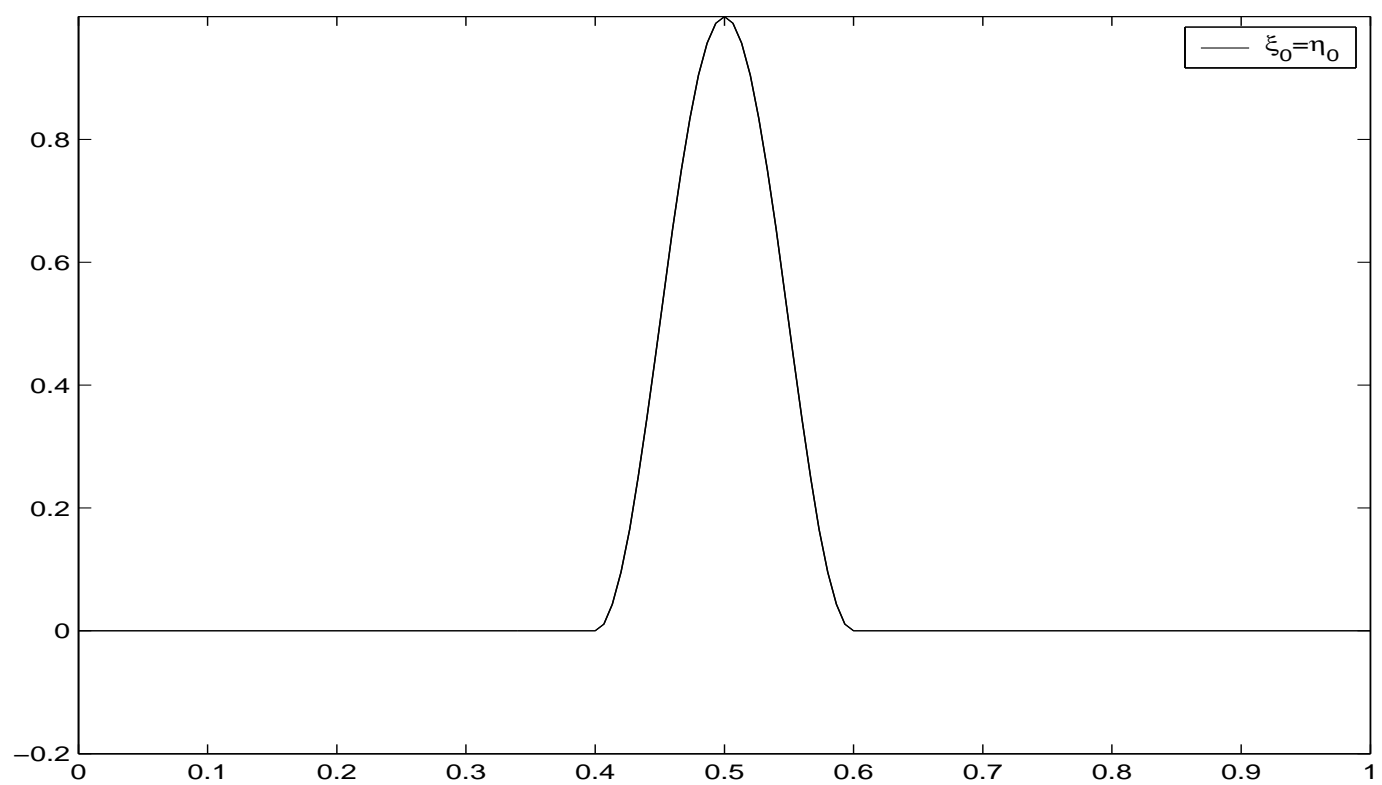

Figure 11 
ROUSSEAU, TEMAM, AND TRIBBIA

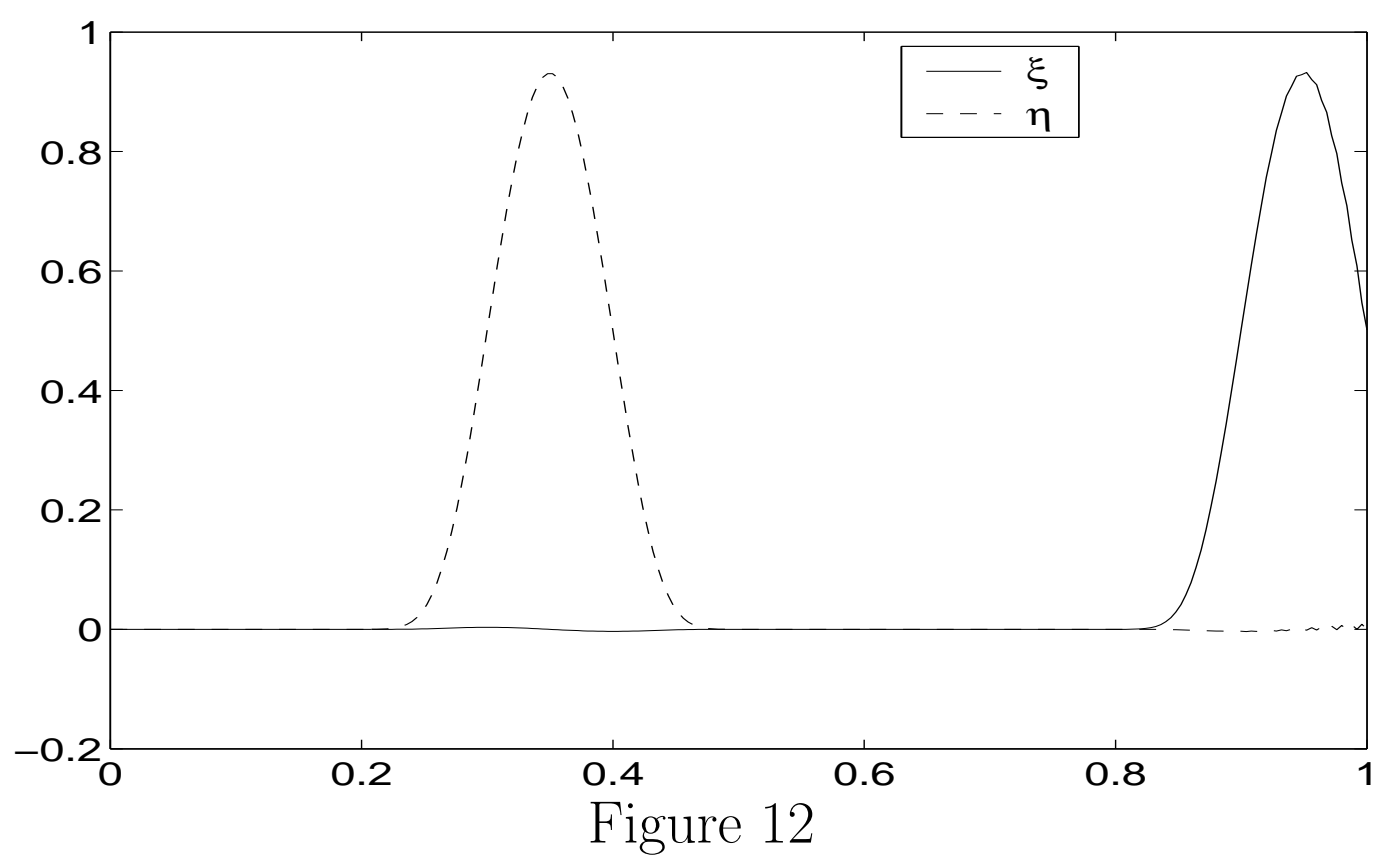


BOUNDARY LAYERS IN AN OCEAN RELATED SYSTEM

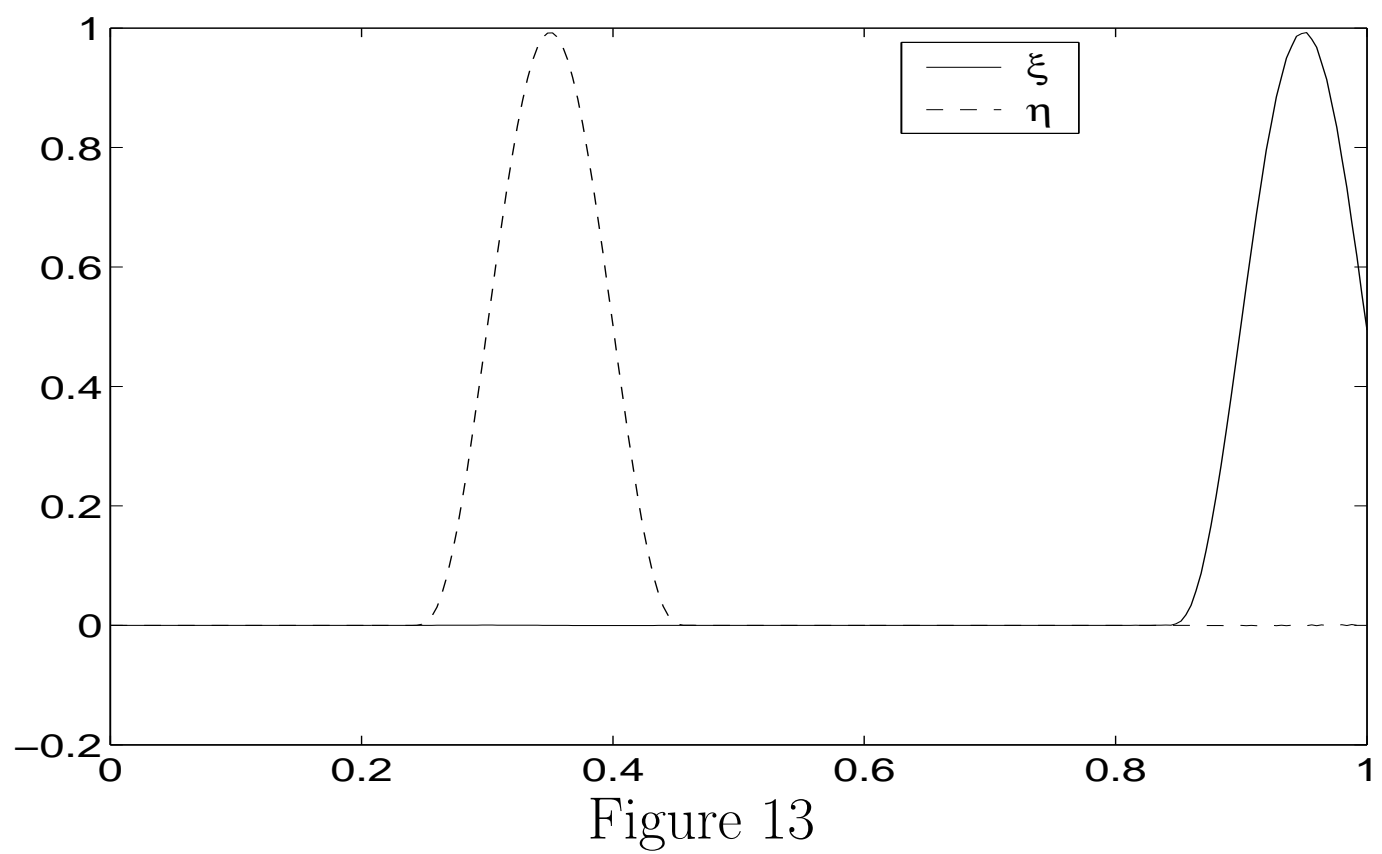


ROUSSEAU, TEMAM, AND TRIBBIA

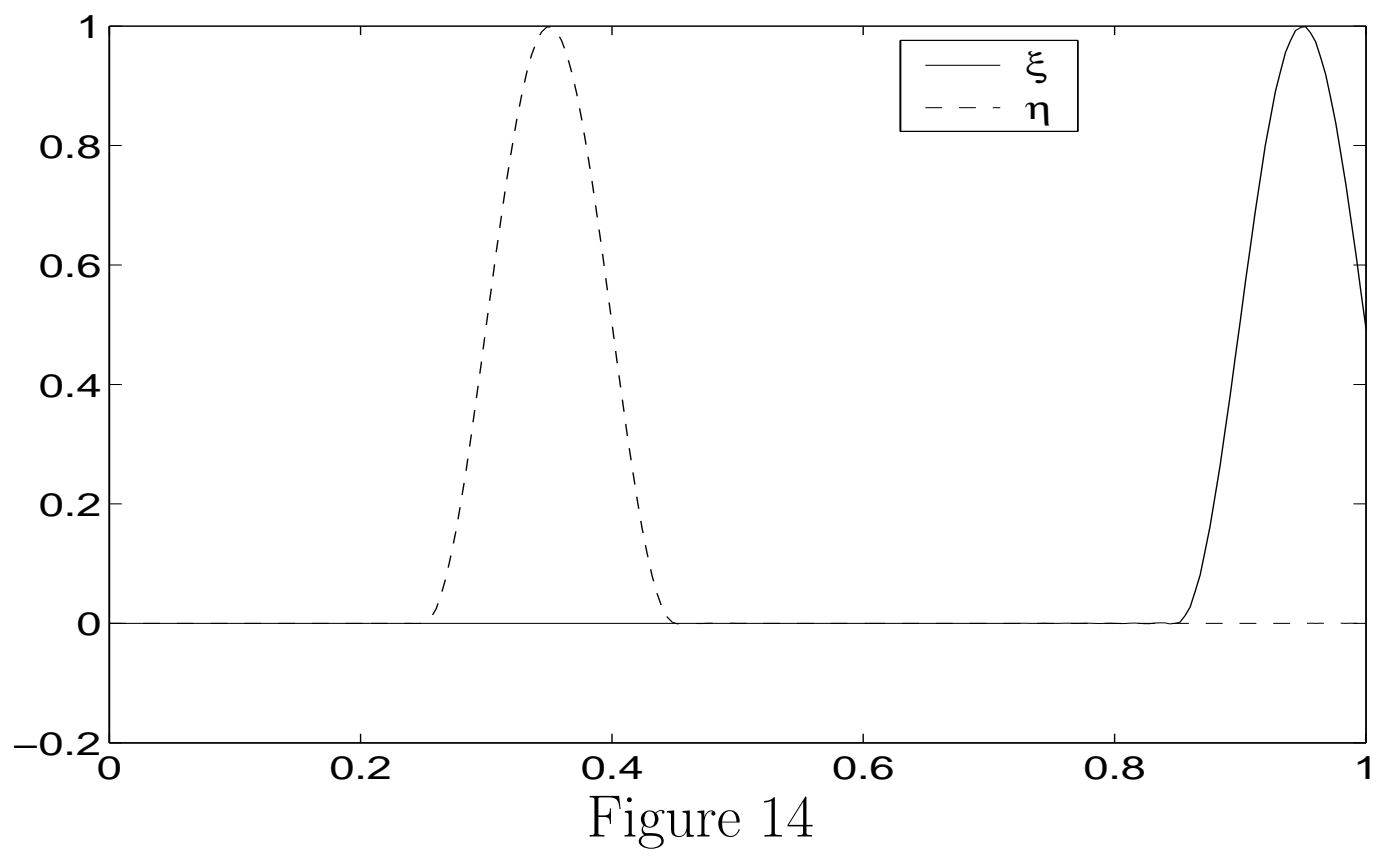


BOUNDARY LAYERS IN AN OCEAN RELATED SYSTEM

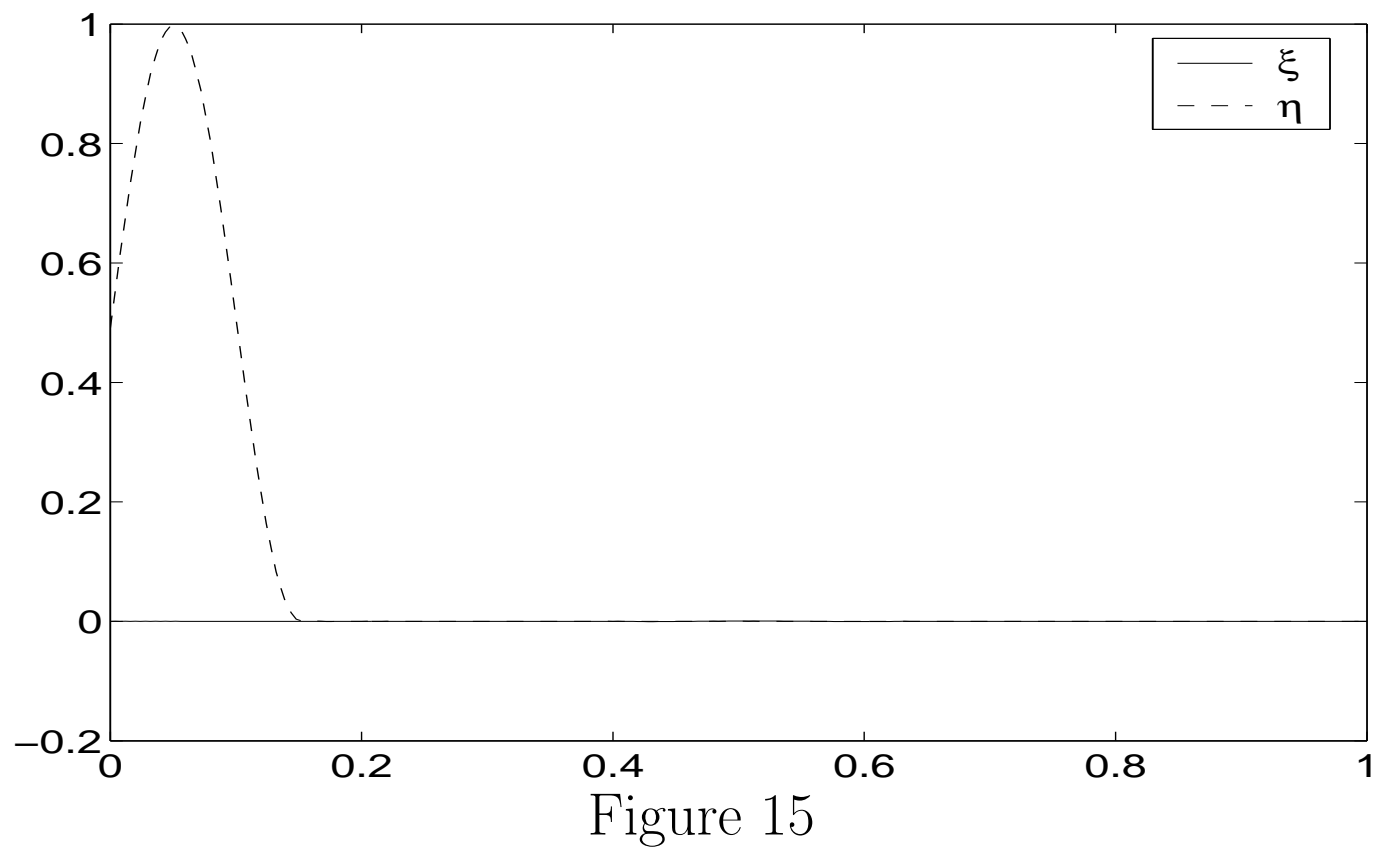


ROUSSEAU, TEMAM, AND TRIBBIA

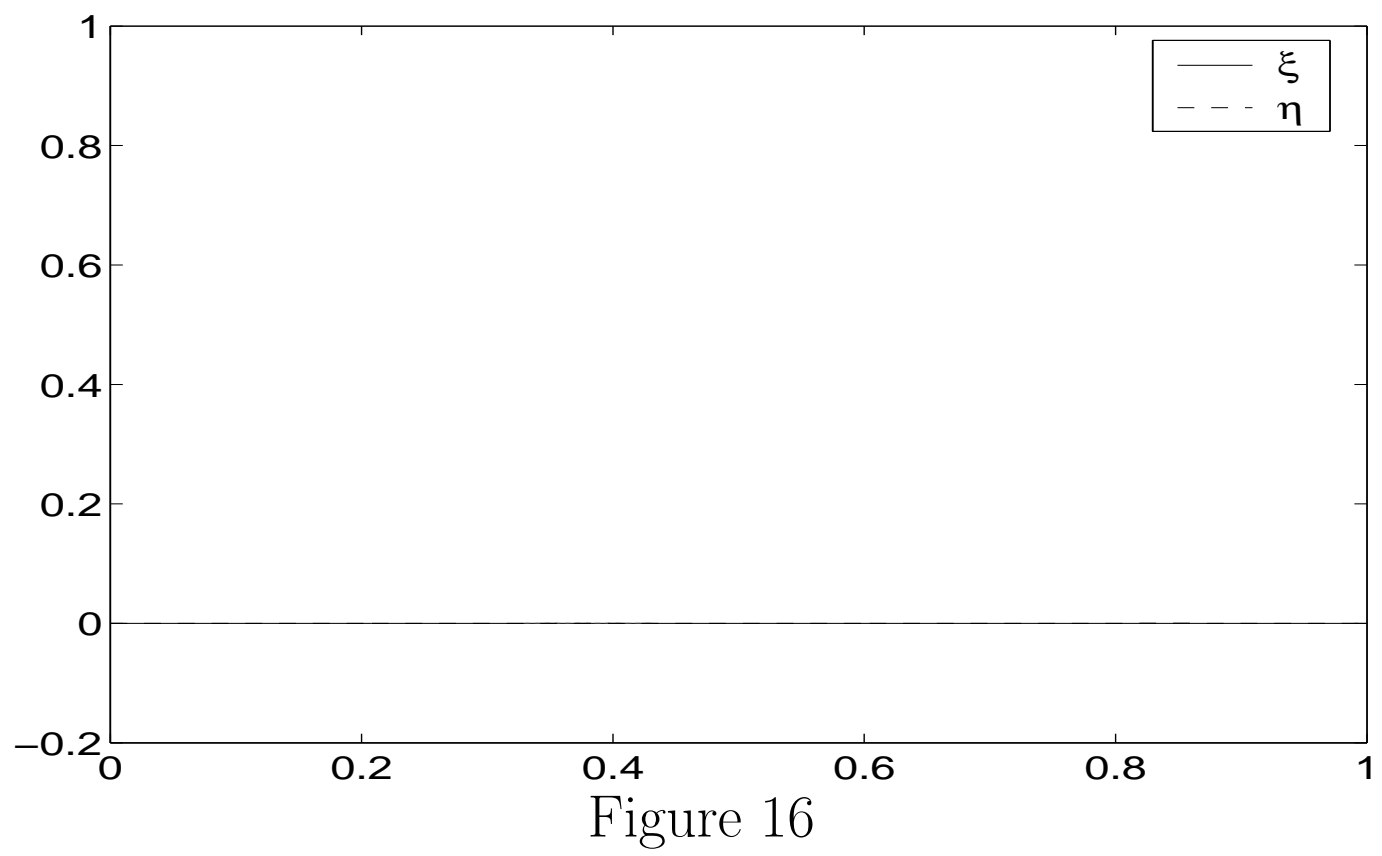


BOUNDARY LAYERS IN AN OCEAN RELATED SYSTEM

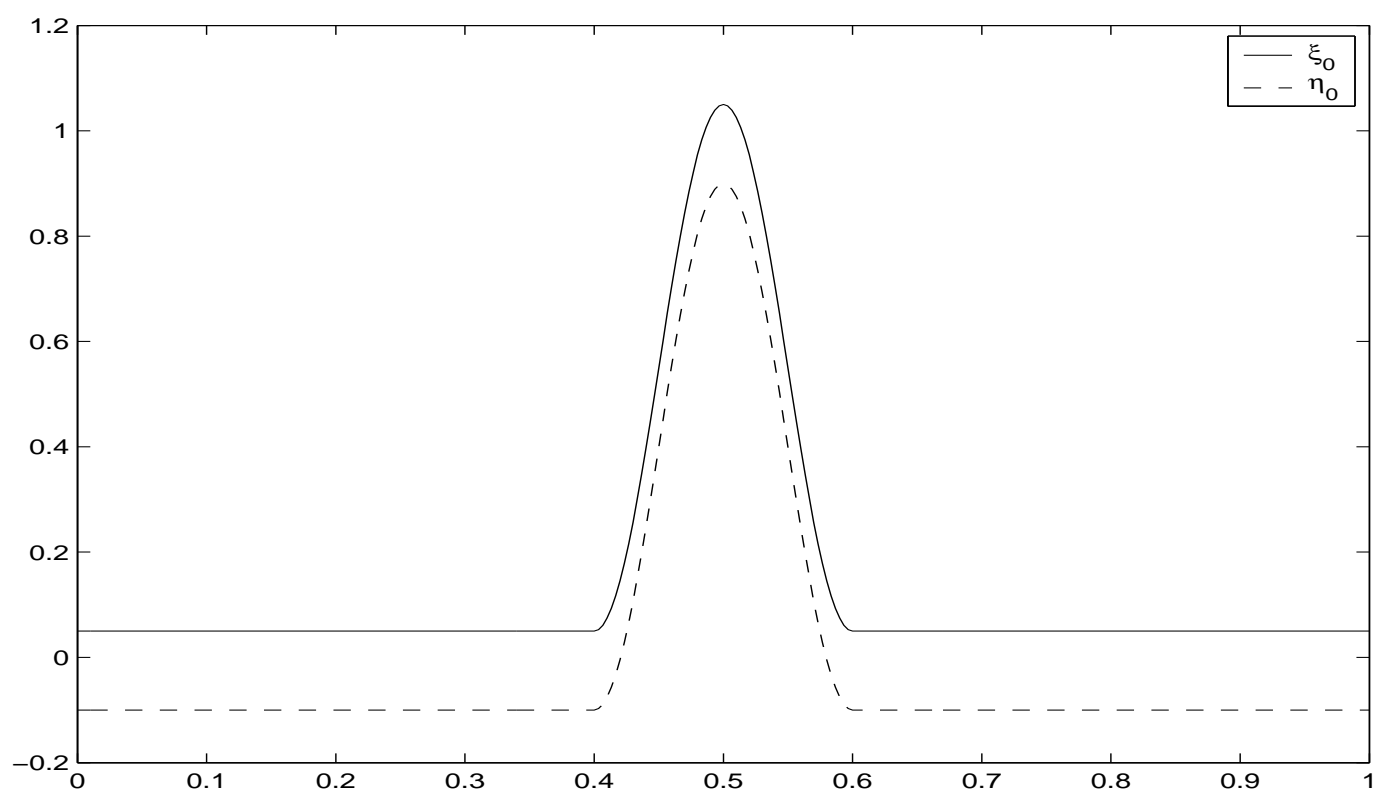

Figure 17 
ROUSSEAU, TEMAM, AND TRIBBIA

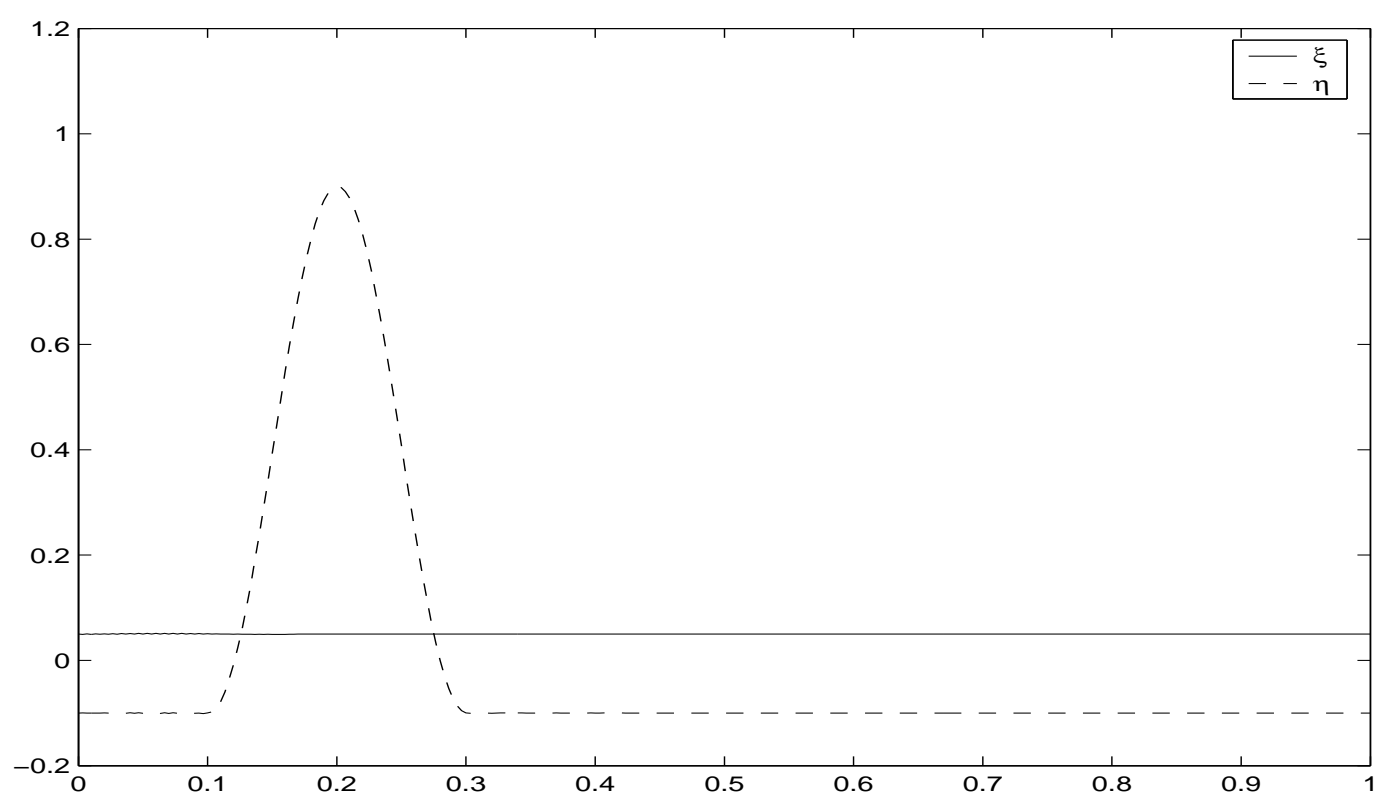

Figure 18 